\title{
Phase Transitions in Materials with Thermal Memory: the Case of Unequal Conductivities
}

John Murrough Golden

Technological University Dublin, Murrough.Golden@TUDublin.ie

Follow this and additional works at: https://arrow.tudublin.ie/scschmatart

Part of the Applied Mathematics Commons, and the Mathematics Commons

\section{Recommended Citation}

Golden, J.M. (2009). Phase transitions in materials with thermal memory: the case of unequal conductivities. Physica D: Nonlinear Phenomena, vol. 238, no. 4, pg. 428-448. doi:10.1016/ j.physd.2008.11.004

This Article is brought to you for free and open access by the School of Mathematics at ARROW@TU Dublin. It has been accepted for inclusion in Articles by an authorized administrator of ARROW@TU Dublin. For more information, please contact arrow.admin@tudublin.ie, aisling.coyne@tudublin.ie,gerard.connolly@tudublin.ie. 


\title{
Phase transitions in materials with thermal memory: The case of unequal conductivities
}

\author{
J.M. Golden* \\ School of Mathematical Sciences, Dublin Institute of Technology, Kevin Street, Dublin 8, Ireland
}

\section{A R T I C L E I N F O}

\section{Article history:}

Received 10 March 2008

Received in revised form

18 September 2008

Accepted 15 November 2008

Available online $\mathrm{xxxx}$

Communicated by Y. Nishiura

\section{PACS:}

64.70.Dv

66.10.Cb

67.40.Pm

65.40.Gr

Keywords:

Materials with thermal memory

Phase transition

Continuum thermodynamics

Asymptotic analysis

Dissipation

Unequal conductivities

\begin{abstract}
A B S T R A C T
A model for thermally induced phase transitions in materials with thermal memory was recently proposed, where the equations determining heatflow were assumed to be the same in both phases. In this work, the model is generalized to the case of phase dependent heatflow relations. The temperature (or coldness) gradient is decomposed into two parts, each zero on one phase and equal to the temperature (or coldness) gradient on the other. However, they vary smoothly over the transition zone. These are treated as separate independent quantities in the derivation of field equations from thermodynamics. Heat flux is given by an integral over the history of the temperature gradient, with different kernels on each phase. Asymptotic analysis is carried out to obtain generalizations of previous results. These involve the jump in temperature across the transition zone and the normal derivatives of the temperature on each phase boundary, which are related to the velocity of the transition zone and a latent heat dependent on this velocity, as well as the speeds of thermal disturbances in the two phases.
\end{abstract}

(C) 2008 Elsevier B.V. All rights reserved.

\section{Introduction}

A model for thermally induced phase transitions in materials with thermal memory was proposed in [1], where the equations determining heat flux were assumed to be the same in both phases. In this work, the model is generalized to the case of phase dependent heat flux relations.

As in [1], the starting point is a general thermodynamic theory of materials with memory. The detailed derivation of the field equations from thermodynamic arguments, applied to a free energy with a quadratic memory dependent term, is omitted, since this derivation is a minor generalization of that outlined in [1]. The independent field variables are the phase field and its gradient, the temperature and the temperature (or coldness) gradient. This last quantity is decomposed into two independent fields, each equal to the temperature or coldness gradient over one phase and zero over the other. The heat flux is given by the sum of contributions involving the history of the temperature gradient with different kernels for each phase.

A short memory limit applied to the order parameter yields relations akin to general results in $[2,3]$ but with phase-dependent thermal memory. The taking of this limit is described in [1] and will be omitted in the present work.

The main motivation for the work reported in [4] is to establish a theory with finite speed of propagation of thermal disturbances. Though the Fourier theory very successfully describes heat movement in conducting bodies, the infinite speed of propagation must be regarded as unphysical

Thus, the viewpoint adopted in [1] and the present work is that the "correct" theory is the one with thermal memory, and the theory based on the Fourier relation is a usually very accurate approximation.

We are considering in this work first order phase transitions (non-zero latent heat) involving solidification (or liquefaction) where different thermal memories are ascribed to the two phases.

\footnotetext{
* Tel.: +353 1 4024611/10; fax: +353 14024994

E-mail address: murrough.golden@dit.ie.
} 
An asymptotic analysis is carried out, both for a simplified model, where the velocity of the transition zone is constant and steady-state conditions have been established, and for the general case. The integro-partial differential equation describing the behaviour of the bulk materials is also discussed.

The results obtained for when the speed of thermal disturbance propagation in each phase is much greater than the normal transition zone speed (in effect, the Fourier limit) show that the temperature field is continuous across the transition zone and the temperature gradients on the phase boundaries obey the Stefan condition.

For transition zone speeds comparable with thermal disturbance speeds, the theory makes some interesting predictions. There is a finite, velocity-dependent discontinuity in the temperature and the temperature gradient fields on the phase boundaries are related to this discontinuity. Also, the ratio of the latent heat to the specific heat must approach zero as the normal transition zone speed approaches the speeds of thermal disturbances.

On the matter of notation, vectors and tensors are denoted by lowercase and uppercase boldface characters respectively and scalars by ordinary script. The real line is denoted by $\mathcal{R}$ and the non-negative reals by $\mathcal{R}^{+}$. The set of tensors acting on $\mathcal{R}^{3}$ is denoted by $\operatorname{Lin}\left(\mathcal{R}^{3}\right)$.

\section{General relations}

We consider a body consisting of two phases, the more viscous phase being referred to as solid and the other as liquid. These are labeled by an order parameter $\varphi$ which takes values 0 at points occupied by the liquid and 1 at points occupied by the solid. It is assumed that there is a transition zone of finite thickness where $\varphi$ takes values in $(0,1)$. Let the body under consideration occupy a volume $B \subset \mathcal{R}^{3}$.

The local absolute temperature at position $\mathbf{x} \in \mathscr{B}$ and time $t$ is $\theta(\mathbf{x}, t) \in \mathcal{R}^{+}$. The heat flux vector is $\mathbf{q}(\mathbf{x}, t) \in \mathcal{R}^{3}$. We introduce a variable $\kappa(\mathbf{x}, t)$ which is the coldness, given by $1 / \theta>0$, and define $\mathbf{g}, \mathbf{d} \in \mathcal{R}^{3}$ as

$$
\mathbf{g}=\nabla \theta, \quad \mathbf{d}=\nabla \kappa=-\frac{1}{\theta^{2}} \mathbf{g}
$$

where $\nabla$ is the gradient with respect to $\mathbf{x}$.

In the formulation of thermodynamics presented in [1], the natural variables are $\boldsymbol{\kappa}$ and $\mathbf{d}$, which is why they are introduced here. However, we switch to $\theta$ and $\mathbf{g}$ below.

The dependence of field quantities on the space variable $\mathbf{x}$ will often be omitted.

The development of the field equations from thermodynamics is identical to that described in [1], except for a generalization which will now be discussed.

Consider a function $h(\phi)$ with the properties

$$
h(1)=1, \quad h(0)=0 .
$$

Also, it is assumed to be invertible on the interval $(0,1)$, so that

$$
h^{\prime}(\varphi)>0
$$

on this interval. One example of such a function is $h(\phi)=\phi^{n}, n>0$. We define

$$
\mathbf{d}_{l}=(1-h) \mathbf{d}, \quad \mathbf{d}_{s}=h \mathbf{d}
$$

so that $\mathbf{d}_{l}$ is equal to $\mathbf{d}$ on the liquid phase and zero on the solid phase. The reverse is true for $\mathbf{d}_{s}$. We have

$$
\mathbf{d}=\mathbf{d}_{l}+\mathbf{d}_{s}
$$

everywhere, including the transition zone. It will be convenient later to use the notation

$$
h_{s}=h, \quad h_{l}=1-h, \quad \text { giving } h_{s}+h_{l}=1 .
$$

Let

$$
\mathbf{l}_{l}(t)=\int_{0}^{t} \mathbf{d}_{l}(u) \mathrm{d} u, \quad \mathbf{l}_{s}(t)=\int_{0}^{t} \mathbf{d}_{s}(u) \mathrm{d} u,
$$

so that

$$
\dot{\mathbf{i}}_{l}(t)=\mathbf{d}_{l}(u), \quad \mathbf{i}_{s}(t)=\mathbf{d}_{s}(u) .
$$

These two field variables replace the variable $\mathbf{l}$ in [1] which is thermodynamically conjugate to the heat flux $\mathbf{q}$. Here, we introduce $\mathbf{q}_{l}, \mathbf{q}_{s}$, thermodynamic conjugates of $\mathbf{l}_{s}, \mathbf{l}_{l}$. The theory can be developed as in [1] with $\mathbf{q} \cdot \mathbf{i}=\mathbf{q} \cdot \mathbf{d}$ replaced by $\mathbf{q}_{s} \cdot \mathbf{i}_{s}+\mathbf{q}_{l} \cdot \mathbf{i}_{l}=\mathbf{q}_{s} \cdot \mathbf{d}_{s}+\mathbf{q}_{l} \cdot \mathbf{d}_{l}$. Thus, there is one more independent and dependent field variable in the theory.

The effect of the splitting of $\mathbf{q}, \mathbf{d}$ into $\mathbf{q}_{s}, \mathbf{q}_{l}, \mathbf{d}_{s}, \mathbf{d}_{l}$ is that the relation for $\mathbf{q}$ in terms of the history of $\mathbf{d}$ is generalized to

$$
\begin{aligned}
& \mathbf{q}_{l}(t)=\int_{0}^{\infty} \mathbf{K}_{l l}(s) \mathbf{d}_{l}^{t}(s) \mathrm{d} s+\int_{0}^{\infty} \mathbf{K}_{l s}(s) \mathbf{d}_{s}^{t}(s) \mathrm{d} s \\
& \mathbf{q}_{s}(t)=\int_{0}^{\infty} \mathbf{K}_{s l}(s) \mathbf{d}_{l}^{t}(s) \mathrm{d} s+\int_{0}^{\infty} \mathbf{K}_{s s}(s) \mathbf{d}_{s}^{t}(s) \mathrm{d} s \\
& \mathbf{K}_{l l}, \mathbf{K}_{l s}, \mathbf{K}_{s l}, \mathbf{K}_{s s}: \mathcal{R}^{+} \mapsto \operatorname{Lin}\left(\mathcal{R}^{3}\right), \\
& \mathbf{d}_{l}^{t}(s)=\mathbf{d}_{l}(t-s), \quad \mathbf{d}_{s}^{t}(s)=\mathbf{d}_{s}(t-s) .
\end{aligned}
$$

The non-diagonal kernels $\mathbf{K}_{l s}, \mathbf{K}_{s l}$ arise from the fact that we allow interactive terms in the free energy between $\mathbf{I}_{s}$ and $\mathbf{I}_{l}$. 
Since we must have $\mathbf{q}_{\mathrm{s}} \cdot \mathbf{d}_{s}+\mathbf{q}_{l} \cdot \mathbf{d}_{l}=\mathbf{q} \cdot \mathbf{d}$ to give the correct form of the first and second laws, it follows that

$$
\mathbf{q}_{l}=\mathbf{q}_{s}=\mathbf{q}
$$

everywhere. Thus

$$
\mathbf{K}_{l l}(u)=\mathbf{K}_{s l}(u)=\mathbf{K}_{l}(u), \quad \mathbf{K}_{s s}(u)=\mathbf{K}_{l s}(u)=\mathbf{K}_{s}(u), \quad s \in \mathcal{R}^{+} .
$$

In terms of the quantities $\mathbf{K}_{l}$ and $\mathbf{K}_{s}$, we have everywhere

$$
\mathbf{q}(\mathbf{x}, t)=\int_{0}^{\infty} \mathbf{K}_{l}(s) \mathbf{d}_{l}^{\mathrm{t}}(\mathbf{x}, s) \mathrm{d} s+\int_{0}^{\infty} \mathbf{K}_{s}(s) \mathbf{d}_{s}^{t}(\mathbf{x}, s) \mathrm{d} s
$$

where the dependence on $\mathbf{x}$ has been explicitly included. Let us assume that the transition zone is moving into the liquid phase (solidification). If $\mathbf{x}$ is in the solid phase at time $t$, the hereditary integrals trace the history of this material point into the transition zone and the liquid phase from the solid phase. If $\mathbf{x}$ is in the liquid phase at time $t$ then the hereditary integral contains $\mathbf{d}_{l}$ evaluated at past times where $\mathbf{x}$ was deeper within this phase. If $\mathbf{x}$ is in the transition zone at time $t$ then the hereditary integrals contain $\mathbf{d}_{l}, \mathbf{d}_{s}$ at past times within the transition zone and $\mathbf{d}_{l}$ in the liquid phase.

As noted earlier, it is convenient, when considering the final field equations, to switch to the temperature $\theta$ and $\mathbf{g}_{s}, \mathbf{g}_{l}$, given by (2.4) with $\mathbf{g}$ replacing $\mathbf{d}$. We introduce the quantity

$$
u=\frac{\theta-\theta_{M}}{\theta_{M}}
$$

which is assumed to be small. The parameter $\theta_{M}$ is the transition temperature.

We assume that heat conduction is isotropic so the conductivity tensors $\mathbf{K}_{l}=K_{l} \mathbf{I}, \mathbf{K}_{s}=K_{s} \mathbf{I}$ where $\mathbf{I}$ is the unit tensor in $\operatorname{Lin}\left(\mathcal{R}^{3}\right)$. Also, referring to (2.1), we write

$$
\mathbf{g}_{l}^{t}(s)=-\theta^{2} \mathbf{d}_{l}^{t}=-\theta^{2} h_{l} \mathbf{d}^{t}, \quad \mathbf{g}_{s}^{t}(s)=-\theta^{2} \mathbf{d}_{s}^{t}=-\theta^{2} h_{s} \mathbf{d}^{t} .
$$

The memory terms in (2.12) are approximated by

$$
-\int_{0}^{\infty} \tilde{k}_{i}(s) \mathbf{g}_{i}^{t}(s) \mathrm{d} s, \quad \tilde{k}_{i}(s)=\frac{K_{i}(s)}{\theta_{M}^{2}}, \quad i=l, s,
$$

where the neglected terms have factors of $O(u)$.

We introduce a double-well potential $v f(\varphi)$ where $v$ is a constant known as the height of the potential and $f$ is a non-negative function with the property that

$$
f(0)=f(1)=0 .
$$

We also introduce a function $g$ obeying

$$
g(1)-g(0)=1 \text {. }
$$

The functions $f$ and $g$ are sometimes chosen to be

$$
f(\varphi)=\frac{1}{2} \varphi^{2}(1-\varphi)^{2}, \quad g(\varphi)=\varphi^{2}(3-2 \varphi) .
$$

This choice of $f$ is the simplest form of a double-well potential with minima at $\varphi=0$ and $\varphi=1$. It obeys (2.16). The choice of $g$ obeys (2.17). It will not be necessary to assign specific forms to these functions.

The casting of the final field equations in dimensionless form is discussed in [1]. These become in the current context. ${ }^{1}$

$$
\begin{aligned}
& \alpha \epsilon^{2} \dot{\varphi}=\epsilon^{2} \Delta \varphi-f^{\prime}(\varphi)-u \beta \epsilon g^{\prime}(\varphi) \\
& \dot{u}=\gamma \dot{g}(\varphi)+\nabla \cdot\left(k_{l} \circ h_{l}^{t} \nabla u^{t}\right)+\nabla \cdot\left(k_{s} \circ h_{s}^{t} \nabla u^{t}\right), \\
& k_{l} \circ h_{l}^{t} \nabla u^{t}=\int_{0}^{\infty} k_{l}(s) h_{l}^{t}(s) \nabla u^{t}(s) \mathrm{d} s=\int_{-\infty}^{t} k_{l}(t-s) h_{l}(s) \nabla u(s) \mathrm{d} s
\end{aligned}
$$

and similarly for $k_{s} \circ h_{s}^{t} \nabla u^{t}$, where $\epsilon^{2} \ll 1$ and $\alpha$ of $O(1)$ are dimensionless parameters. Also

$$
\beta \epsilon=\frac{\lambda}{v}, \quad \gamma=\frac{\lambda}{\theta_{M} c}
$$

where $\lambda$ is the latent heat and $c$ the specific heat, which is assumed to be constant. The quantity $\lambda$ is defined in [1] as the difference between the internal energy in the liquid phase at the transition temperature, adjacent to the transition zone and that in the solid phase. The functions $k_{l}, k_{s}$ are the dimensionless versions of $\tilde{k}_{l}, \tilde{k}_{s}$, given by $(2.15)_{2}[1]$.

\footnotetext{
1 Note that $f^{\prime}(\varphi)+u \beta \in g^{\prime}(\varphi)$ in (2.19) vanishes at $\varphi=0,1$. Therefore, these are stationary points of the free energy introduced in [1], where space and time dependence of $\varphi$ are neglected. In fact, using (2.18), one can show that for $u>-1 /(6 \beta \epsilon)$ the stationary point $\varphi=0$, corresponding to the liquid phase, is a minimum while, for $u<1 /(6 \beta \epsilon)$ the point $\varphi=1$ is a minimum [5]. Thus, the liquid phase can exist for $u<0$ and the solid phase for $u>0$.
} 
The heat flux (2.12), for the approximation (2.15), is given in dimensionless form by

$$
\mathbf{q}(t)=-\int_{0}^{\infty} k_{l}(s) h_{l}^{t}(s) \nabla u^{t}(s) \mathrm{d} s-\int_{0}^{\infty} k_{s}(s) h_{s}^{t}(s) \nabla u^{t}(s) \mathrm{d} s .
$$

The speeds of heat disturbance propagation are given in dimensionless form by [1].

$$
V_{l}=\sqrt{k_{l}(0)}, \quad V_{s}=\sqrt{k_{s}(0)} .
$$

There would seem to be a physical argument for assuming that $V_{s}>V_{l}$. However, we will leave this issue open in what follows.

The Fourier limit will be of interest later. This is obtained by putting

$$
k_{l}(s)=K_{l} \mu \mathrm{e}^{-\mu s}, \quad k_{s}(s)=K_{s} \mu \mathrm{e}^{-\mu s},
$$

where $K_{l}, K_{s}$ are the material conductivities in the liquid and solid phases respectively, giving

$$
k_{i}(0)=V_{i}^{2}=K_{i} \mu, \quad k_{i}^{\prime}(0)=-K_{i} \mu^{2}, \quad i=l, s
$$

and taking the large $\mu$ limit.

The transition zone $\mathcal{Z}$ is defined as

$$
\mathcal{Z}=\{\mathbf{x}: \varphi(\mathbf{x}, t, \epsilon) \in(0,1)\} .
$$

Consider the surfaces

$$
\Sigma_{p}(t, \epsilon)=\{\mathbf{x}: \varphi(\mathbf{x}, t, \epsilon)=p\}, \quad p \in(0,1) .
$$

The limits $p \rightarrow 0^{+}, 1^{-}$yield the borders of the transition zone $\mathcal{Z}$. We also choose a surface $\Sigma(t)$ within $Z$ as a reference interface. The exact choice of this surface is not crucial to later arguments. It will be defined by $(2.26)$ with $p=\frac{1}{2}$ and $\varphi(\mathbf{x}, t, \epsilon)=\varphi(\mathbf{x}, t, 0)=\varphi_{0}(\mathbf{x}, t)$.

To avoid proliferation of notation, we shall denote $h_{i}(\varphi(\mathbf{x}, t)), i=l, s$ with different arguments as convenient in a given context, keeping the same functional label. Examples include $h_{i}(s)$ where the time variable $s$ indicates the total time dependence of $h_{i}$, omitting space dependence. Also, we may indicate space (or space and time) dependence but omit $\varphi$. Similar licence will be taken with the temperature variable $u$ - though of course in this case, there is no (necessary) intermediate functional dependence on $\varphi$.

\section{Steady-state case}

Let us first consider a simplified model ([6] for example) where the transition zone is moving at constant dimensionless velocity.

$$
-\mathbf{V}=-\left(V_{x}, V_{y}, V_{z}\right), \quad V_{x}>0
$$

and steady-state conditions have been established. The solid, for which $\varphi=1$, is on the positive side and the liquid, for which $\varphi=0$, is on the negative side. Thus

$$
\varphi(\mathbf{x}, t)=\varphi\left(x+V_{x} t, y+V_{y} t, z+V_{z} t\right), \quad u(\mathbf{x}, t)=u\left(x+V_{x} t, y+V_{y} t, z+V_{z} t\right) .
$$

We write (2.19) in a frame moving with the transition zone:

$$
\begin{aligned}
& \alpha \epsilon^{2} \mathbf{V} \cdot \nabla \varphi=\epsilon^{2} \Delta \varphi-f^{\prime}(\varphi)-u \beta \epsilon \mathrm{g}^{\prime}(\varphi) \\
& \mathbf{V} \cdot \nabla u=\gamma \mathbf{V} \cdot \nabla g(\varphi)+\nabla \cdot \int_{0}^{\infty} k_{l}(s) h_{l}(\mathbf{x}-\mathbf{V} s) \nabla u(\mathbf{x}-\mathbf{V} s) \mathrm{d} s+\nabla \cdot \int_{0}^{\infty} k_{s}(s) h_{s}(\mathbf{x}-\mathbf{V} s) \nabla u(\mathbf{x}-\mathbf{V} s) \mathrm{d} s,
\end{aligned}
$$

where

$$
\phi=\phi(\mathbf{x}), \quad h_{i}=h_{i}(\mathbf{x})=h_{i}(\varphi(\mathbf{x})), \quad i=l, s, \quad u=u(\mathbf{x}),
$$

in terms of the quantities on the right of (3.2) 1,2 . Let us assume that the reference interface, which, in the present section, we refer to as $\&$, rather than $\Sigma(t)$, goes through the origin. We choose the coordinate axis so that, at the origin, the $x$ direction is normal to $\delta$ and the $y z$ plane is tangential to this surface.

Near the origin, $\&$ can be approximated by a paraboloid described by the equation

$$
x-\frac{y^{2}}{2 R_{y}}-\frac{z^{2}}{2 R_{z}}=0
$$

where $R_{y}, R_{z}$ are the radii of curvature at the origin in the $x y, x z$ planes respectively, once the $y$ and $z$-axes have been chosen to be the principal directions of the paraboloid. For $R_{y}, R_{z}$ positive, $\delta$ curves into the positive $x$ halfspace.

Along a line through $\mathbf{x}$, parallel and close to the $x$-axis, let the transition zone $\mathcal{Z}$ occupy the interval

$$
\begin{aligned}
& x_{-}(y, z) \leq x^{\prime} \leq x_{+}(y, z), \quad x_{-}<0, \quad x_{+}>0 \\
& x_{ \pm}(y, z)=x_{ \pm}(0,0)-\frac{y^{2}}{2 R_{y}}-\frac{z^{2}}{2 R_{z}} .
\end{aligned}
$$

The transition zone $\mathcal{Z}$ near the origin can be visualized as a set of almost parallel surfaces of the form ( 3.5$)$ but with a constant $x_{0}$, rather than zero on the right, where

$$
x_{-}(0,0) \leq x_{0} \leq x_{+}(0,0) .
$$


On each surface, $\phi$ has a constant value. The surface $\phi=f_{0}$, where $f_{0}$ is a constant, through $x_{0}$ can be written as

$$
\begin{aligned}
\varphi(x, y, z) & =\varphi\left(x_{0}, 0,0\right)=\varphi\left(x-\frac{y^{2}}{2 R_{y}}-\frac{z^{2}}{2 R_{z}}, 0,0\right) \\
& \approx \varphi(x, 0,0)-\varphi_{x}(x, 0,0)\left(\frac{y^{2}}{2 R_{y}}+\frac{z^{2}}{2 R_{z}}\right)
\end{aligned}
$$

in the vicinity of the origin. This form applies to all choices of $x_{0}$ so we take

$$
\varphi(x, y, z)=\varphi(x, 0,0)-\varphi_{x}(x, 0,0)\left(\frac{y^{2}}{2 R_{y}}+\frac{z^{2}}{2 R_{z}}\right)
$$

as the approximate form of $\varphi$ in the vicinity of the origin for $\mathbf{x} \in \mathcal{Z}$, though noting the issue that $\varphi_{x}$ is large across the transition zone. Here and below, coordinate variables as subscripts denote differentiation with respect to that variable, except where indicated otherwise. ${ }^{2} \mathrm{~A}$ surface of constant $\phi$ will closely parallel that for constant $u$, since the phase transition is temperature driven. This will be exactly true to lowest order; see (3.53). Applying the arguments in (3.8) gives a form similar to (3.9):

$$
u(x, y, z)=u(x, 0,0)-u_{x}(x, 0,0)\left(\frac{y^{2}}{2 R_{y}}+\frac{z^{2}}{2 R_{z}}\right) .
$$

We decompose the integral in $(3.3)_{2}$ as follows for $\mathbf{x}$ in the transition zone:

$$
\int_{0}^{\infty} k_{i}(s) \nabla \cdot\left(h_{i}(\mathbf{x}-\mathbf{V} s) \nabla u(\mathbf{x}-\mathbf{V} s)\right) \mathrm{d} s=\int_{0}^{s_{-}} k_{i}(s) \nabla \cdot\left(h_{i}(\mathbf{x}-\mathbf{V} s) \nabla u(\mathbf{x}-\mathbf{V} s)\right) \mathrm{d} s+\int_{s_{-}}^{\infty} k_{i}(s) \Delta u(\mathbf{x}-\mathbf{V} s) \mathrm{d} s, \quad i=l, s,
$$

where $s_{-}(\mathbf{x})>0$ is such that $\mathbf{x}-\mathbf{V} s_{-}$is on the negative boundary of $Z$. In fact, it obeys the equation

$$
s_{-}=\frac{1}{V_{x}}\left(x-x_{-}\left(y-V_{y} s_{-}, z-V_{z} s_{-}\right)\right) .
$$

We are interested only in points close to the $x$-axis. Neglecting the squares of lateral quantities in (3.6) 2 gives

$$
s_{-}=\frac{1}{V_{x}}\left(x-x_{-}(0,0)\right) \text {. }
$$

The dimensionless time $s_{-}$is of order $O(\epsilon)$. Also, $s_{-}$depends on $\epsilon$ by virtue of (2.26). We shall use the dominant version of this quantity (that associated with $\epsilon=0$ ) and show in the appropriate places that the correction due to higher orders is negligible.

Observe that the final term on the right of (3.11) is non-zero only if $i=l$.

For $\mathbf{x}=(x, y, z)$ in $Z$ we replace $x$ by

$$
r=\frac{x}{\epsilon}
$$

Let

$$
\begin{aligned}
& u(r, y, z)=u_{0}(r, y, z)+\epsilon u_{1}(r, y, z)+O\left(\epsilon^{2}\right) \\
& \varphi(r, y, z)=\varphi_{0}(r, y, z)+\epsilon \varphi_{1}(r, y, z)+O\left(\epsilon^{2}\right)
\end{aligned}
$$

inside the transition zone, and

$$
\bar{u}(x, y, z)=\bar{u}_{0}(x, y, z)+\epsilon \bar{u}_{1}(x, y, z)+O\left(\epsilon^{2}\right)
$$

outside of $Z$. In this latter region

$$
\bar{\varphi}(x, y, z)=\bar{\varphi}_{0}(x, y, z)+\epsilon \bar{\varphi}_{1}(x, y, z)+O\left(\epsilon^{2}\right)= \begin{cases}1, & x>0 \\ 0, & x<0 .\end{cases}
$$

Putting $\epsilon=0$, we have

$$
\bar{\varphi}_{0}(x, y, z)= \begin{cases}1, & x>0 \\ 0, & x<0\end{cases}
$$

for $\bar{\varphi}_{0}$ so that

$$
\bar{\varphi}_{i}(x, y, z)=0, \quad i=1,2 \ldots .
$$

The matching condition on $u$ is

$$
u(x / \epsilon, y, z)=u(r, y, z)=\bar{u}(\epsilon r, y, z)
$$

\footnotetext{
2 The notation $V_{x}, V_{y}, V_{z}$, indicating components of velocity, is an exception to the rule where subscripts indicate differentiation, as is $R_{y}, R_{z}$ for the radii of curvature.
} 
for large $r$ and small $\epsilon r$. More precisely, this relation holds for $\epsilon r$ in the vicinity of $x_{+}$and $x_{-}$defined in (3.6), near the origin. Putting $\epsilon=0$, we obtain

$$
u_{0}( \pm \infty, y, z)=\bar{u}_{0}(0 \pm, y, z) .
$$

Observe that, from (3.20), we have

$$
u_{r}(r, y, z)=\epsilon \bar{u}_{x}(\epsilon r, y, z), \quad u_{\pi}(r, y, z)=\epsilon^{2} \bar{u}_{x x}(\epsilon r, y, z),
$$

and similarly for higher derivatives. Relation (3.22) and (3.15) yield

$$
\begin{array}{ll}
u_{0 r}( \pm \infty, y, z)=0, & u_{1 r}( \pm \infty, y, z)=\bar{u}_{0 x}(0 \pm, y, z), \\
u_{0 r r}( \pm \infty, y, z)=0, & u_{1 r r}( \pm \infty, y, z)=0 .
\end{array}
$$

The functions $\bar{u}_{0}(0 \pm, y, z), \bar{u}_{0 x}(0 \pm, y, z)$ are the boundary values of these quantities on the positive and negative sides of $z$. Note that from (3.22),

$$
u_{0 r}(r, x, y)=O(\epsilon), \quad u_{0 r r}(r, x, y)=O\left(\epsilon^{2}\right) \quad u_{1 r r}(r, x, y)=O(\epsilon)
$$

for $\epsilon r$ in the vicinity of $x_{ \pm}$. The matching conditions on $\varphi$ are

$$
\begin{aligned}
& \varphi(r, y, z)=1 \quad r \gg 1, \quad \varphi(r, y, z)=0 \quad r \ll-1, \\
& \varphi_{r}(r, y, z)=\varphi_{y}(r, y, z)=\varphi_{z}(r, y, z)=0, \quad|r| \gg 1,
\end{aligned}
$$

yielding

$$
\begin{aligned}
& \varphi_{0}(\infty, y, z)=1, \quad \varphi_{0}(-\infty, y, z)=0 \quad \varphi_{i}( \pm \infty, y, z)=0, \quad i=1,2, \ldots \\
& \varphi_{i r}( \pm \infty, y, z)=\varphi_{i y}( \pm \infty, y, z)=\varphi_{i z}( \pm \infty, y, z)=0, \quad i=0,1, \ldots
\end{aligned}
$$

and similarly for higher derivatives.

\subsection{Asymptotic analysis of the order parameter equation}

To leading order, $(3.3)_{1}$ becomes, in the transition zone,

$$
\varphi_{0 r r}-f^{\prime}\left(\varphi_{0}\right)=0
$$

subject to the boundary conditions

$$
\varphi_{0}(-\infty, y, z)=0, \quad \varphi_{0}(+\infty, y, z)=1, \quad \varphi_{0}(0, y, z)=\frac{1}{2},
$$

recalling the discussion after (2.26). The solution has the explicit form, for $f$ given by $(2.18)_{1}$,

$$
\varphi_{0}(r, y, z)=\frac{1}{2}\left(1+\tanh \left(\frac{r}{2}\right)\right)=\varphi_{0}(r) .
$$

The quantity $\varphi_{0}$ depends only on $r$. The $O(\epsilon)$ terms of $(3.3)_{1}$ yield the relation

$$
\varphi_{1 r r}-f^{\prime \prime}\left(\varphi_{0}\right) \varphi_{1}=u_{0} \beta g^{\prime}\left(\varphi_{0}\right)+\alpha V_{x} \varphi_{0 r}+\mathcal{K}_{0} \varphi_{0 r}
$$

where $\mathcal{K}_{0}$ is given by

$$
\mathcal{K}_{0}=\frac{1}{R_{y}}+\frac{1}{R_{y}} .
$$

A solvability condition on this equation gives the modified Gibbs-Thomson boundary condition [1]

$$
u_{a v}=-\frac{\sigma}{\lambda}\left(\alpha V_{x}+\mathcal{K}_{0}\right)
$$

where

$$
u_{a v}=\int_{-\infty}^{\infty} u_{0}\left(r^{\prime}\right) g_{r}\left(\varphi_{0}\left(r^{\prime}\right)\right) \mathrm{d} r^{\prime}
$$

noting

$$
\int_{-\infty}^{\infty} g_{r}\left(\varphi_{0}\left(r^{\prime}\right)\right) \mathrm{d} r^{\prime}=1
$$

and $\sigma$ is the surface tension. The quantity $u_{a v}$ is the average of the (possibly) strongly varying $u_{0}$ over the transition zone, weighted by $g_{r}$. For $g$ given by $(2.18)_{2}$, the quantity $g_{r}$ is non-negative, goes to zero for large $|r|$, has a maximum in $\mathcal{Z}$ at $\varphi_{0}=\frac{1}{2}$ or $r=0$ and is symmetric about the maximum. ${ }^{3}$ We will give general formulae for $u_{a v}$ in the next section.

$\overline{3}$ Indeed, one can show that $g_{r}\left(\varphi_{0}\right)=12 f\left(\varphi_{0}\right)$, where $f$ is given by $(2.18)_{1}$. 
3.2. Asymptotic analysis of the heat equation

Relation $(3.3)_{2}$ is now multiplied by $\epsilon^{2}$. It is assumed that $k_{s}, k_{l}$ do not vary strongly over times of order $s_{-}$. Consider the first term on the right of (3.11). We have to $O\left(\epsilon^{2}\right)$, noting (3.14),

$$
\begin{aligned}
\epsilon^{2} & \int_{0}^{s_{-}} k_{i}(s) \nabla \cdot\left(h_{i} \nabla u\right)\left(r-V_{x} \frac{s}{\epsilon}, y-V_{y} s, z-V_{z} s\right) \mathrm{d} s=\epsilon^{3} \int_{0}^{s_{-} / \epsilon} k_{i}\left(\epsilon s^{\prime}\right) \nabla \cdot\left(h_{i} \nabla u\right)\left(r-V_{x} s^{\prime}, y-\epsilon V_{y} s^{\prime}, z-\epsilon V_{z} s^{\prime}\right) \mathrm{d} s^{\prime} \\
= & \frac{\epsilon}{V_{x}}\left\{k _ { i } ( 0 ) \int _ { r _ { - } } ^ { r } \left[\left(h_{i 0}\left(r^{\prime}\right) u_{0 r}\left(r^{\prime}\right)\right)_{r}+\epsilon\left(h_{i 1}\left(r^{\prime}\right) u_{0 r}\left(r^{\prime}\right)+h_{i 0}\left(r^{\prime}\right) u_{1 r}\left(r^{\prime}\right)\right)_{r}-\epsilon \frac{\left(r-r^{\prime}\right)}{V_{x}}\left(V_{y}\left(h_{i 0}\left(r^{\prime}\right) u_{0 r}\left(r^{\prime}\right)\right)_{r y}+V_{z}\left(h_{i 0}\left(r^{\prime}\right) u_{0 r}\left(r^{\prime}\right)\right)_{r z}\right)\right.\right. \\
& \left.\left.+\epsilon^{2} h_{i 0}\left(r^{\prime}\right) \Delta_{2} u_{0}\left(r^{\prime}\right)\right] \mathrm{d} r^{\prime}+\epsilon \frac{k_{i}^{\prime}(0)}{V_{x}} \int_{r_{-}}^{r}\left(r-r^{\prime}\right)\left(h_{i 0}\left(r^{\prime}\right) u_{0 r}\left(r^{\prime}\right)\right)_{r} \mathrm{~d} r^{\prime}\right\} \quad i=l, s, r_{-}=r-V_{x} \frac{s_{-}}{\epsilon}=\frac{1}{\epsilon} x_{-}(0,0),
\end{aligned}
$$

where the fact that, by virtue of (3.29),

$$
h_{i 0}\left(r^{\prime}\right)=h_{i}\left(\varphi_{0}\left(r^{\prime}\right)\right), \quad i=l, s
$$

are independent of $y, z$ has been used. Note that, from (2.2), (2.6) and (3.26), we have

$$
\begin{aligned}
& h_{s 0}(\infty)=1, \quad h_{s 0}(-\infty)=0 \\
& h_{10}(\infty)=0, \quad h_{l 0}(-\infty)=1 .
\end{aligned}
$$

The quantities

$$
u_{0}\left(r^{\prime}\right)=u_{0}\left(r^{\prime}, y, z\right), \quad h_{i 1}\left(r^{\prime}\right)=h_{i}^{\prime}\left(\varphi_{0}\left(r^{\prime}\right)\right) \varphi_{1}\left(r^{\prime}, y, z\right), \quad i=l, s
$$

depend on $y, z$. The prime on $h_{i}$ indicates differentiation with respect to $\varphi_{0}$. Relation (3.13) has been used and

$$
\Delta_{2}=\frac{\partial}{\partial y^{2}}+\frac{\partial}{\partial z^{2}}
$$

By virtue of (3.10), the term with $\Delta_{2}$ is $O\left(\epsilon^{2}\right)$. Also, to $O\left(\epsilon^{2}\right)$

$$
\epsilon^{2} \mathbf{V} \cdot \nabla u-\epsilon^{2} \gamma \mathbf{V} \cdot \nabla g(\varphi)=\epsilon V_{x} u_{0 r}-\epsilon \gamma V_{x} g_{r}\left(\varphi_{0}\right)+\epsilon^{2}\left[V_{x} u_{1 r}+V_{y} u_{0 y}+V_{z} u_{0 z}-V_{x} \gamma\left(g^{\prime}\left(\varphi_{0}\right) \varphi_{1}\right)_{r}\right],
$$

where terms $g_{y}\left(\varphi_{0}\right)$ and $g_{z}\left(\varphi_{0}\right)$ do not contribute, since $\varphi_{0}$ depends only on $r$.

We first consider terms of $O(\epsilon)$ in (3.3) 2 multiplied by $\epsilon^{2}$. The second term on the right of (3.11) multiplied by $\epsilon^{2}$ gives a contribution of $O\left(\epsilon^{2}\right)$ and can be neglected. We therefore need only include the terms of $O(\epsilon)$ in (3.35) and (3.40). The integration of the term from (3.35) can be carried out and we obtain

$$
v_{x}^{2} u_{0 r}-V_{x}^{2} \gamma g_{r}\left(\varphi_{0}\right)-\left(k_{l}(0) h_{l 0}+k_{s}(0) h_{s 0}\right) u_{0 r}=0
$$

where all field quantities are evaluated at $r$. The quantity $u_{0 r}\left(r_{-}, y, z\right)$ is $O(\epsilon)$ by (3.24) and is neglected. On integrating over $(-\infty, \infty)$, we obtain, recalling (2.17) and (2.22),

$$
\begin{aligned}
& \left(V_{x}^{2}-V_{s}^{2}\right) u_{0}(\infty)-\left(V_{x}^{2}-V_{l}^{2}\right) u_{0}(-\infty)+\left(V_{s}^{2}-V_{l}^{2}\right) u_{a}=V_{x}^{2} \gamma, \\
& u_{a}(y, z)=\int_{-\infty}^{\infty} h_{s r}\left(\varphi_{0}\left(r^{\prime}\right)\right) u_{0}\left(r^{\prime}\right) \mathrm{d} r^{\prime},
\end{aligned}
$$

where (3.26) and (3.37) have been used, together with

$$
h_{10 r}(r)=-h_{s 0 r}(r) \text {, }
$$

which follows from (2.6). We introduce the notation:

$$
V_{ \pm}^{2}=\frac{1}{2}\left(V_{s}^{2} \pm V_{l}^{2}\right)
$$

and

$$
\left[u_{0}\right]_{ \pm}=u_{0}(\infty, y, z) \pm u_{0}(-\infty, y, z)=\bar{u}_{0}(0+, y, z) \pm \bar{u}_{0}(0-, y, z)=\left[\bar{u}_{0}\right]_{ \pm}
$$

where (3.21) has been used.

In order to determine $u_{a}$, let us consider the solution of (3.41) in more detail. Applying (2.6) to lowest order, we can write (3.41) as

$$
H\left(\varphi_{0}(r)\right) u_{0 r}(r)=V_{x}^{2} \gamma g_{r}\left(\varphi_{0}(r)\right), \quad H\left(\varphi_{0}(r)\right)=V_{x}^{2}-V_{l}^{2}-2 V_{-}^{2} h_{s}\left(\varphi_{0}(r)\right)
$$

Let us put

$$
h_{s}(\varphi)=h(\varphi), \quad \mathrm{g}(\varphi(h))=A(h), \quad H(\varphi)=B(h(\varphi)) .
$$

The invertibility of $h(\varphi)$ on $(0,1)$ has been used in defining $A(h)$. This quantity, which is the function $g$ applied to $h^{-1}$, expresses the connection between $h(\varphi)$ and $g(\varphi)$. The simplest connection is of course that they are the same function, giving $A(h)=h$. We shall however leave $A$ unspecified in subsequent developments. Relation (3.46) can be written as

$$
u_{0 r}(r)=V_{x}^{2} \gamma E_{r}\left(h\left(\varphi_{0}(r)\right)\right)
$$


where

$$
E(h)=\int_{0}^{h} \frac{A^{\prime}(\xi)}{B(\xi)} \mathrm{d} \xi=-\frac{1}{2 V_{-}^{2}} \int_{0}^{h} \frac{A^{\prime}(\xi)}{\xi-w} \mathrm{~d} \xi, \quad w=\frac{V_{x}^{2}-V_{l}^{2}}{V_{s}^{2}-V_{l}^{2}} .
$$

We have

$$
E^{\prime}(h)=\frac{A^{\prime}(h)}{B(h)}, \quad A^{\prime}(h)=\frac{\mathrm{d} g}{\mathrm{~d} \varphi}\left[\frac{\mathrm{d} h}{\mathrm{~d} \varphi}\right]^{-1} \geq 0 .
$$

If $A(h)=h$ then

$$
E(h)=-\frac{1}{2 V_{-}^{2}} \log \left|\frac{w-h}{w}\right| .
$$

Defining

$$
V_{m}=\min \left(V_{s}, V_{l}\right), \quad V_{M}=\max \left(V_{s}, V_{l}\right),
$$

we see that for $V_{x}<V_{m}$, the function $B(h)$ is negative and non-zero when $h \in[0,1]$. For $V_{x}>V_{M}$ the function $B(h), h \in[0,1]$ is positive. It is not clear that $V_{x}$ can exceed either $V_{s}$ or $V_{l}$ or both. However, we do not exclude the possibility. For $V_{x} \in\left[V_{m}, V_{M}\right]$, we have $0 \leq w \leq 1$, the quantity $B(h)$ changes sign in the interval $[0,1]$ and is zero at $h=w$. In this case, we take the integral defining $E(h)$ in $(3.50)$ to be the principal value if $h>w$.

The fact that $B(w)$ vanishes means that $H\left(\varphi_{0}(r)\right)$ in (3.46) is equal to zero at a certain point (dependent on the value of $\left.V_{x}^{2}\right)$ in the transition zone. Since the function $g_{r}$ on the right-hand side need not vanish at this point, $u_{0 r}(r)$ will generally have a singularity at the point $r \in Z$ corresponding to $h=w \in[0,1]$.

The quantity $A(h)$ cannot depend on $w$ because this would imply that the functions $h(\varphi)$ and/or $g(\varphi)$ depend on a parameter relating to a specific configuration of the system, while they were introduced into the theory as general field variables, equally applicable to any configuration. The field quantity $\varphi$ will of course acquire such dependence on specific configuration parameters as a result of the solution of the problem, but this is not true of the functional forms $h$ and $g$. Thus, $E(h)$ must depend on $w$, because $A^{\prime}(\xi)$ in (3.49) cannot cancel such dependence in $B(\xi)$.

It is of some interest to note that if $A(h)$ were allowed to have a dependence on $w$ then other possibilities would emerge, including one where $J$ is independent of $w$. This would lead to consequences quite different to those derived below regarding the position of singularities.

Relation (3.48) gives

$$
u_{0}(r)=V_{x}^{2} \gamma E\left(h\left(\varphi_{0}(r)\right)\right)+u_{0}(-\infty),
$$

and in particular

$$
\left[u_{0}\right]_{-}=V_{x}^{2} \gamma E(1) \text {. }
$$

The quantities $u_{a}, u_{a v}$ defined by $(3.42)_{2}$ and (3.33) respectively, have the form

$$
\begin{aligned}
& u_{a}=V_{x}^{2} \gamma \int_{0}^{1} E(\xi) \mathrm{d} \xi+u_{0}(-\infty), \\
& u_{a v}=V_{x}^{2} \gamma \int_{0}^{1} E(\xi) A^{\prime}(\xi) \mathrm{d} \xi+u_{0}(-\infty) .
\end{aligned}
$$

We note that, with the aid of (3.54), $u_{a}$ can be written as

$$
\begin{aligned}
& u_{a}=J u_{0}(\infty)+(1-J) u_{0}(-\infty)=\frac{1}{2}\left(\left[u_{0}\right]_{+}-(1-2 J)\left[u_{0}\right]_{-}\right) \\
& =J \bar{u}_{0}(0+)+(1-J) \bar{u}_{0}(0-)=\frac{1}{2}\left(\left[\bar{u}_{0}\right]_{+}-(1-2 J)\left[\bar{u}_{0}\right]_{-}\right), \\
& J=\frac{\int_{0}^{1} E(\xi) \mathrm{d} \xi}{E(1)}=J(w) \text {. }
\end{aligned}
$$

From (3.49) and (3.50), we see that the function $E(\xi) / E(1)$ is non-negative and less than or equal to unity for $V_{x}<V_{m}$ and for $V_{x}>V_{M}$. By the mean value theorem, it follows that

$$
J \in(0,1), \quad V_{x}<V_{m}, \quad V_{x}>V_{M} .
$$

For $V_{x}^{2} \in\left[V_{m}^{2}, V_{M}^{2}\right], J$ varies over $(-\infty, \infty)$.

Partial integration in the numerator of (3.56) 5 and some further manipulations yield that

$$
J=1-w+\frac{1}{2 V_{-}^{2} E(1)},
$$

where $A(0)=0$ and $A(1)=1$ have been used. All quantities can be determined explicitly for polynomial and other choices of $A(h)$. Using (3.56), we can write (3.42) in the form

$$
\left[u_{0}\right]_{-}=\left[\bar{u}_{0}\right]_{-}=\frac{V_{x}^{2} \gamma}{V_{x}^{2}-V_{J}^{2}}, \quad V_{J}^{2}=V_{s}^{2}(1-J)+V_{l}^{2} J
$$


which generalizes a result in [1]. Comparing this with (3.54), we see that

$$
E(1)=\frac{1}{V_{x}^{2}-V_{J}^{2}}
$$

which is consistent with (3.58). Thus, the solutions of the relation $V_{x}^{2}=V_{J}^{2}$ correspond to the singularities of $E(1)$ as a function of $V_{x}^{2}$. The quantity

$$
-2 V_{-}^{2} E(1)=\int_{0}^{1} \frac{A^{\prime}(\xi)}{\xi-w} \mathrm{~d} \xi
$$

diverges logarithmically ([9] for example) as $w \rightarrow 0$ or 1 , corresponding to $V_{x}^{2} \rightarrow V_{l}^{2}$ or $V_{s}^{2}$. This implies that

$$
J \rightarrow 1, \quad V_{J}^{2} \rightarrow V_{l}^{2} \text { as } V_{x}^{2} \rightarrow V_{l}^{2} \quad \text { while } J \rightarrow 0, \quad V_{J}^{2} \rightarrow V_{s}^{2} \text { as } V_{x}^{2} \rightarrow V_{s}^{2} .
$$

The quantity $E(1)$ has no other singularity so that the non-linear equation

$$
V_{x}^{2}=V_{s}^{2}(1-J(w))+V_{l}^{2} J(w)
$$

has two solutions as described in (3.62). The right-hand side of $(3.59)_{2}$ is singular in these two limits. To avoid a divergence in [ $\left.\bar{u}_{0}\right]_{-}$we must assume that $\gamma=\gamma(\mathbf{V})$ and that this quantity vanishes at least as strongly as $\left[\log \left(V_{x}^{2}-V_{l}^{2}\right)\right]^{-1},\left[\log \left(V_{x}^{2}-V_{s}^{2}\right)\right]^{-1}$ for $V_{x} \rightarrow V_{l}, V_{s}$ respectively, which generalizes an analogous observation in [1].

For $V_{x}^{2}<V_{J}^{2}$ and $\gamma$ positive, relation (3.59) gives that $\left[\bar{u}_{0}\right]_{-}$is negative.

For $V_{x}^{2} \ll V_{J}^{2}$, which occurs in particular in the Fourier limit given by (2.23), the discontinuity in the temperature field given by (3.59) is negligible.

Relation $(3.55)_{2}$ gives that

$$
u_{a v}=\frac{1}{2}\left[u_{0}\right]_{+}+\left[u_{0}\right]_{-}\left\{\frac{\int_{0}^{1} E(\xi) A^{\prime}(\xi) \mathrm{d} \xi}{E(1)}-\frac{1}{2}\right\},
$$

by virtue of (3.54). The second term vanishes as $V_{-}^{2} \rightarrow 0$, since by (3.46), (3.47) and (3.49), $E(h)$ is proportional to $A(h)$ in this limit, while $A(1)=1$, so that (3.64) reduces to a result given in [1] for the equal conductivities case.

In the light of this analysis, we can address the question of the physical significance, if any, of the choice of $h=h_{s}$. It is clear that this choice affects the physical result obtained, in particular, the form of $\left[\bar{u}_{0}\right]_{-}$, through the parameter $J$. This is determined by the function $A(h)$ in $(3.47)_{2}$, which specifies the relationship between $g(\varphi)$ and $h(\varphi)$. It is presumably a matter for experiment to decide on the correct choice of $A(h)$.

The simplest choice would seem to be to take $g, h$ to be the same function so that, as noted earlier, $A(h)=h$. Recalling (3.51) and (3.58), we see that this yields

$$
J=\frac{V_{s}^{2}-V_{x}^{2}}{V_{s}^{2}-V_{l}^{2}}-\left\{\log \left|\frac{V_{s}^{2}-V_{x}^{2}}{V_{l}^{2}-V_{x}^{2}}\right|\right\}^{-1} .
$$

Let us now consider $O\left(\epsilon^{2}\right)$ terms in $(3.3)_{2}$ multiplied by $\epsilon^{2}$, that is, terms of $O(1)$ in the original relation. The overall factor $\epsilon^{2}$ will be omitted when discussing the various terms. Firstly, we examine various terms in (3.35). Partial integration gives that

$$
\int_{r_{-}}^{r}\left(r-r^{\prime}\right) \frac{\partial}{\partial r^{\prime}}\left[h_{i 0}\left(r^{\prime}\right) u_{0 r}\left(r^{\prime}, y, z\right)\right] \mathrm{d} r^{\prime}=-\left(r-r_{-}\right) h_{i 0}\left(r_{-}\right) u_{0 r}\left(r_{-}, y, z\right)+\int_{r_{-}}^{r} h_{i 0}\left(r^{\prime}\right) u_{0 r}\left(r^{\prime}, y, z\right) \mathrm{d} r^{\prime}, \quad i=l, s,
$$

where $h_{i 0}\left(r^{\prime}\right)$ is an abbreviation of $h_{i}\left(\varphi_{0}\left(r^{\prime}\right)\right)$. By virtue of $(3.24)_{1}$, the first term on the right of $(3.66)$ can be neglected and the second term gives

$$
h_{i 0}(r) u_{0}(r, y, z)-h_{i 0}\left(r_{-}\right) u_{0}\left(r_{-}, y, z\right)-\int_{r_{-}}^{r} h_{i 0 r}\left(r^{\prime}\right) u_{0}\left(r^{\prime}, y, z\right) \mathrm{d} r^{\prime}, \quad i=l, s .
$$

Also,

$$
\int_{r_{-}}^{r}\left(r-r^{\prime}\right) \frac{\partial}{\partial r^{\prime}}\left[h_{i 0}\left(r^{\prime}\right) u_{0 r y}\left(r^{\prime}, y, z\right)\right] \mathrm{d} r^{\prime}=h_{i 0}(r) u_{0 y}(r, y, z)-h_{i 0}\left(r_{-}\right) u_{0 y}\left(r_{-}, y, z\right)-\int_{r_{-}}^{r}\left[h_{i 0 r}\left(r^{\prime}\right) u_{0 y}\left(r^{\prime}, y, z\right)\right] \mathrm{d} r^{\prime}, \quad i=l, s
$$

and similarly for the term with $u_{0 r z}$. Furthermore, from (3.10) and (3.14), we have

$$
\begin{aligned}
\epsilon \int_{r_{-}}^{r} h_{i 0}\left(r^{\prime}\right) \Delta_{2} u_{0}\left(r^{\prime}, y, z\right) \mathrm{d} r^{\prime} & =-\mathcal{K}_{0} \int_{r_{-}}^{r} h_{i 0}\left(r^{\prime}\right) u_{0 r}\left(r^{\prime}, y, z\right) \mathrm{d} r^{\prime} \\
& =-\mathcal{K}_{0}\left\{\left(h_{i 0}(r) u_{0}(r, y, z)-h_{i 0}\left(r_{-}\right) u_{0}\left(r_{-}, y, z\right)\right)-\int_{r_{-}}^{r} h_{i 0 r}\left(r^{\prime}\right) u_{0}\left(r^{\prime}, y, z\right) \mathrm{d} r^{\prime}\right\}
\end{aligned}
$$

where $\mathcal{K}_{0}$ is given by (3.31). We have replaced $u_{0 r}(r, 0,0)$ by $u_{0 r}(r, y, z)$ on the right of $(3.69)_{1}$. These two quantities are almost equal for small $y, z$ by virtue of $(3.10)$. 
The contribution to (3.35) due to a second order correction in $s_{-}$, namely $s_{-} \rightarrow s_{-}+\epsilon \eta$, where $\eta$ is $O(\epsilon)$, has the form (see the first term on the right of $(3.35)_{2}$ without the factor $\left.\epsilon^{2}\right)$

$$
\frac{1}{V_{x} \epsilon} \eta k_{i}(0)\left(h_{i 0} u_{0 r}\right)_{r}\left(r_{-}, y, z\right)
$$

which is negligible by (3.24) and (3.25).

We take the difference between quantities of $O(1)$ in (3.3) (not multiplied by $\epsilon^{2}$ ) at $r= \pm \infty$, or in the case of the last term in (3.11) (non-zero only if $i=l$ ), the difference between this expression across $\mathcal{Z}$, which is given by

$$
D=\int_{s_{-}\left(\mathbf{x}_{+}\right)}^{\infty} k_{l}(s) \Delta \bar{u}_{0}\left(\mathbf{x}_{+}-\mathbf{V} s\right) \mathrm{d} s-\int_{0}^{\infty} k_{l}(s) \Delta \bar{u}_{0}\left(\mathbf{x}_{-}-\mathbf{V} s\right) \mathrm{d} s
$$

where $\mathbf{x}_{ \pm}$are on the boundaries of $\mathcal{Z}$ as specified by (3.6). There is no discontinuity for quantities evaluated outside of $\mathcal{Z}$, so we have

$$
D=-\int_{0}^{s_{-}\left(\mathbf{x}_{+}\right)} k_{l}(s) \Delta \bar{u}_{0}\left(\mathbf{x}_{-}-\mathbf{V} s\right) \mathrm{d} s .
$$

Now $s_{-}\left(\mathbf{x}_{+}\right)$is of order $\epsilon$ so that $D$ is $O(\epsilon)$ and may be neglected.

Also, terms independent of $r$ cancel. This includes all terms evaluated at $r_{-}$which is independent of $r$ by virtue of the last relation of (3.35). One such term is that arising out of the integration of the dominant contribution to $(3.35)_{2}$, which was neglected in deriving (3.41), as noted after that equation. It must be included among second order terms. Also, the integrals in (3.67) and (3.68) can be taken as being over $\mathcal{R}$ for $r=\infty$ and are equal to $u_{a}(y, z)$, defined by $(3.42)_{2}$, or its derivatives if $i=s$. For $i=l$, by (3.43), they are equal to the negative of these quantities. At $r=-\infty$, they can be neglected. Thus, on using (3.21), (3.23), (3.26), (3.35), (3.40) and (3.67)-(3.69), we obtain

$$
\begin{aligned}
& \left(V_{x}^{2}-V_{s}^{2}\right) u_{1 r}(\infty)-\left(V_{x}^{2}-V_{l}^{2}\right) u_{1 r}(-\infty)=\left(V_{x}^{2}-V_{s}^{2}\right) \bar{u}_{0 x}(0+)-\left(V_{x}^{2}-V_{l}^{2}\right) \bar{u}_{0 x}(0-) \\
& =\frac{1}{V_{x}}\left\{-\left(V_{x}^{2}+V_{s}^{2}\right)\left(u_{0 y}(\infty, y, z) V_{y}+u_{0 z}(\infty, y, z) V_{z}\right)+\left(V_{x}^{2}+V_{l}^{2}\right)\left(u_{0 y}(-\infty, y, z) V_{y}+u_{0 z}(-\infty, y, z) V_{z}\right)\right. \\
& \left.\quad+\left(V_{s}^{2}-V_{l}^{2}\right)\left(u_{a y}(y, z) V_{y}+u_{a z}(y, z) V_{z}\right)+C_{s}(0) u_{0}(\infty, y, z)-C_{l}(0) u_{0}(-\infty, y, z)-\left(C_{s}(0)-C_{l}(0)\right) u_{a}(y, z)\right\}
\end{aligned}
$$

where

$$
C_{i}(s)=k_{i}^{\prime}(s)-V_{x} \mathcal{K}_{0} k_{i}(s), \quad i=l, s .
$$

With the aid of (3.56) and (3.59) 2 , this can be put in the form

$$
\begin{aligned}
\left(V_{x}^{2}-V_{s}^{2}\right) \bar{u}_{0 x}(0+)-\left(V_{x}^{2}-V_{l}^{2}\right) \bar{u}_{0 x}(0-) & =\left(V_{x}^{2}-V_{+}^{2}\right)\left[\bar{u}_{0 x}\right]_{-}-V_{-}^{2}\left[\bar{u}_{0 x}\right]_{+} \\
& =\frac{1}{V_{x}}\left\{-\left(V_{x}^{2}+V_{J}^{2}\right)\left(V_{y}\left[\bar{u}_{0}\right]_{y-}+V_{z}\left[\bar{u}_{0}\right]_{z-}\right)+C_{J}(0)\left[u_{0}\right]_{-}\right\}
\end{aligned}
$$

where notation from (3.44) and (3.45) has been used, together with

$$
C_{J}(\tau)=(1-J) C_{s}(\tau)+J C_{l}(\tau)
$$

while $\left[\bar{u}_{0}\right]_{-}$is given by (3.59) and we have put $\left[\bar{u}_{0 y}\right]_{ \pm}=\left[\bar{u}_{0}\right]_{y \pm},\left[\bar{u}_{0 z}\right]_{ \pm}=\left[\bar{u}_{0}\right]_{z \pm}$. Since $\mathbf{V}$ is assumed in the present model to be independent of $\mathbf{x}$, the derivatives of the first term on the right of (3.59) with respect to $y$ and $z$ vanishes. Thus, we have

$$
\left(V_{x}^{2}-V_{s}^{2}\right) \bar{u}_{0 x}(0+)-\left(V_{x}^{2}-V_{l}^{2}\right) \bar{u}_{0 x}(0-)=\frac{1}{V_{x}} C_{J}(0)\left[\bar{u}_{0}\right]_{-}=\frac{C_{J}(0) V_{x} \gamma(\mathbf{V})}{V_{x}^{2}-V_{J}^{2}},
$$

on using (3.59).

Relations (3.77) is different from the the Stefan condition connecting the discontinuity in the temperature gradient (and the heat flux) to the velocity of the transition zone, which holds in the Fourier case, though this latter form is recovered from (3.77) by taking the short memory limit described by (2.23) and related comments.

Relation (3.77) gives that

$$
\begin{aligned}
& \left.\bar{u}_{0 x}(0-)\right|_{V_{x}=V_{s}}=-\frac{\left.C_{J}(0)\left[\bar{u}_{0}\right]_{-}\right|_{V_{x}=V_{s}}}{V_{s}\left(V_{s}^{2}-V_{l}^{2}\right)} \\
& \left.\bar{u}_{0 x}(0+)\right|_{V_{x}=V_{s}}=\left.\frac{\mathrm{d}}{\mathrm{d} V_{x}^{2}} F_{s}\left(V_{x}\right)\right|_{V_{x}=V_{s}},
\end{aligned}
$$

where

$$
F_{s}\left(V_{x}\right)=\left(V_{x}^{2}-V_{l}^{2}\right) \bar{u}_{0 x}(0-)+\frac{1}{V_{x}} C_{J}(0)\left[\bar{u}_{0}\right]_{-}
$$

and

$$
\begin{aligned}
& \left.\bar{u}_{0 x}(0+)\right|_{V_{x}=V_{l}}=-\frac{C_{J}(0)\left[\bar{u}_{0}\right]-\mid v_{x}=V_{l}}{V_{l}\left(V_{s}^{2}-V_{l}^{2}\right)} \\
& \left.\bar{u}_{0 x}(0-)\right|_{V_{x}=V_{l}}=\left.\frac{\mathrm{d}}{\mathrm{d} V_{x}^{2}} F_{l}\left(V_{x}\right)\right|_{V_{x}=V_{l}},
\end{aligned}
$$


where

$$
F_{l}\left(V_{x}\right)=\left(V_{x}^{2}-V_{s}^{2}\right) \bar{u}_{0 x}(0+)-\frac{1}{V_{x}} C_{J}(0)\left[\bar{u}_{0}\right]_{-} .
$$

The usefulness of $(3.78)_{2}$ and $(3.80)_{2}$ is limited by the fact that we do not know the dependence of the individual $\bar{u}_{0 x}(0 \pm)$ on $V_{x}^{2}$. However, certain conclusions can be drawn from them. Note that the differentiation of (3.79) and (3.81) with respect to $V_{x}^{2}$ produces a factor $\left(V_{x}^{2}-V_{J}^{2}\right)^{-2}\left(\mathrm{~d} J / \mathrm{d} V_{x}^{2}\right)$ in one term respectively from the rightmost contributions to these relations, those dependent on $\left[\bar{u}_{0}\right]_{-}$. This is clear from the rightmost expression in (3.77).

Thus, the comment after (3.63) can be strengthened. The quantity $\gamma(\mathbf{V})$ must go to zero for $V_{x} \rightarrow V_{l}$ or $V_{s}$ at least as strongly as $\left[\log \left(V_{x}^{2}-V_{l}^{2}\right)\right]^{-2}\left(V_{x}^{2}-V_{l}^{2}\right),\left[\log \left(V_{x}^{2}-V_{s}^{2}\right)\right]^{-2}\left(V_{x}^{2}-V_{s}^{2}\right)$ in these two cases, since the derivative of $J(w)$ diverges linearly at the end points. This generalizes a conclusion in [1]. It furthermore yields that the right-hand sides of $(3.78)_{1}$ and $(3.80)_{1}$ vanish.

Relations (3.59) and (3.77) will apply to the liquefaction (rather than solidification) process by changing the sign of $V_{x}$. Thus, in (3.59), the sign of $\left[\bar{u}_{0}\right]_{-}$is unchanged while if $\mathcal{K}_{0}$ in (3.77) is replaced by $-\mathcal{K}_{0}$, then this relation changes sign.

Therefore, $\left[\bar{u}_{0}\right]_{-}$is negative for both solidification and liquefaction. In the former case, the process is driven by low temperatures in the solid phase and temperatures in the vicinity of $u=0$ in the liquid phase. For liquefaction, it would be driven by high temperatures in the liquid phase and temperatures around $u=0$ in the solid phase. The greater the temperature differences, the more rapid the process in both cases, as implied by (3.59) for values of $V_{x}<V_{m}$, the latter given by (3.52) and $V_{x}$ not too close to $V_{m}$, if we assume that the variation of $\gamma(\mathbf{V})$ with $V_{x}$ is small until $V_{x}$ approaches $V_{m}$.

\subsection{The heat equation outside $\mathcal{Z}$}

Let us now consider (3.3) 2 outside of $\mathcal{Z}$, temporarily dropping the notation $\bar{u}$ for dimensionless temperature fields outside the transition zone. In the liquid phase (which, in fixed coordinates, is ablating) we have simply

$$
\mathbf{V} \cdot \nabla u=\int_{0}^{\infty} k_{l}(s) \Delta u(\mathbf{x}-\mathbf{V} s) \mathrm{d} s=\Delta \int_{0}^{\infty} k_{l}(s) u(\mathbf{x}-\mathbf{V} s) \mathrm{d} s
$$

and the heat flux equation (2.21) is given by

$$
\mathbf{q}(\mathbf{x})=-\int_{0}^{\infty} k_{l}(s) \nabla u(\mathbf{x}-\mathbf{V} s) \mathrm{d} s .
$$

For $\mathbf{x}=(x, y, z)$ in the solid phase, the situation is more complicated. Let the quantity $\tau(\mathbf{x})$ be the time period elapsed since the surface $\delta$ passing through the origin, crossed $\mathbf{x}$. Also, $\tau_{ \pm}$are the limits to $\tau$ from above and below. The surface $\&$ is taken to be the interface between the phases, at which the discontinuities occur. We seek to neglect the thickness of the interface in this discussion, though it will prove necessary to consider transition zone structure for a specific purpose. Referring to (3.5), one sees that the curve $\tau(\mathbf{x})-\tau\left(\mathbf{x}_{0}\right)=0$ for $\mathbf{x}$ variable (but in the vicinity of $\mathrm{x}_{0}$, the point which maps into the origin on $\S$ ), can be approximated by the curve

$$
x-V_{x} \tau\left(\mathbf{x}_{0}\right)-\frac{\left(y-V_{y} \tau\left(\mathbf{x}_{0}\right)\right)^{2}}{2 R_{y}}-\frac{\left(z-V_{z} \tau\left(\mathbf{x}_{0}\right)\right)^{2}}{2 R_{z}}=0
$$

where the squared terms are small. Thus, we have

$$
\tau(\mathbf{x})=\frac{1}{V_{x}}\left\{x-\frac{\left(y-V_{y} \tau\left(\mathbf{x}_{0}\right)\right)^{2}}{2 R_{y}}-\frac{\left(z-V_{z} \tau\left(\mathbf{x}_{0}\right)\right)^{2}}{2 R_{z}}\right\} .
$$

Taking the gradient and neglecting terms with factors $y-V_{y} \tau, z-V_{z} \tau$, we obtain

$$
\nabla \tau \approx \frac{1}{V_{x}}(1,0,0) \text {. }
$$

Also

$$
\Delta \tau(\mathbf{x}) \approx-\frac{\mathcal{K}_{0}}{V_{x}}
$$

where $\mathcal{K}_{0}$ is given by (3.31).

Let $k: \mathcal{R}^{+} \mapsto \mathcal{R}$ be defined by

$$
\begin{aligned}
k(s) & =k_{l}(s), & & s>\tau(\mathbf{x}) \\
& =k_{s}(s), & & s<\tau(\mathbf{x}) .
\end{aligned}
$$

We can write (2.21) as

$$
\mathbf{q}(\mathbf{x})=-\int_{0}^{\infty} k(s) \nabla u(\mathbf{x}-\mathbf{V} s) \mathrm{d} s .
$$

Both $k(s)$ and $u(\mathbf{x}-\mathbf{V} s)$ are discontinuous at $s=\tau(\mathbf{x})$. Observe that

$$
\mathbf{V} \cdot \mathbf{q}(\mathbf{x})=-\int_{0}^{\infty} k(s) \mathbf{V} \cdot \nabla u(\mathbf{x}-\mathbf{V} s) \mathrm{d} s=\int_{0}^{\infty} k(s) \frac{\partial}{\partial s} u(\mathbf{x}-\mathbf{V} s) \mathrm{d} s .
$$




\section{ARTICLE IN PRESS}

The discontinuity in $u(\mathbf{x}-\mathbf{V} s)$ will give singular delta function behaviour at $s=\tau$, yielding a contribution

$$
\left(u\left(\mathbf{x}-\mathbf{V} \tau_{+}\right)-u\left(\mathbf{x}-\mathbf{V} \tau_{-}\right)\right) \int_{\tau_{1}}^{\tau_{2}} k(s) \delta(s) \mathrm{d} s, \quad \tau \in\left(\tau_{1}, \tau_{2}\right) .
$$

The integral is however indeterminate because $k$ has a discontinuity at $s=\tau$. It can yield any value between $k_{s}(\tau)$ and $k_{l}(\tau)$. We can write this contribution as

$$
\left(u\left(\mathbf{x}-\mathbf{V} \tau_{+}\right)-u\left(\mathbf{x}-\mathbf{V} \tau_{-}\right)\right)\left(k_{s}(\tau)(1-U)+k_{l}(\tau) U\right), \quad U \in(0,1) .
$$

This difficulty was not present in [1]. In order to determine $U$, we need to revert temporarily to a finite thickness model of $Z$. We restrict the discussion to $V_{x}<V_{m}$, defined by (3.52). This ensures in particular that $J<1$ as observed in (3.57). Relation (2.21) becomes

$$
\begin{aligned}
& \mathbf{q}=-\int_{0}^{\tau_{-}(\mathbf{x})} k_{s}(s) \nabla u(\mathbf{x}-\mathbf{V} s) \mathrm{d} s-\int_{\tau_{+}(\mathbf{x})}^{\infty} k_{l}(s) \nabla u(\mathbf{x}-\mathbf{V} s) \mathrm{d} s-\mathbf{I}\left(\tau_{-}, \tau_{+}\right), \\
& \mathbb{I}\left(\tau_{-}, \tau_{+}\right)=\int_{\tau_{-}(\mathbf{x})}^{\tau_{+}(\mathbf{x})}\left[k_{s}(s) h_{s}(\mathbf{x}-\mathbf{V} s) \nabla u(\mathbf{x}-\mathbf{V} s)+k_{l}(s) h_{l}(\mathbf{x}-\mathbf{V} s) . \nabla u(\mathbf{x}-\mathbf{V} s)\right] \mathrm{d} s .
\end{aligned}
$$

Let

$$
\mathbf{X}=(X, Y, Z)=\mathbf{x}-\mathbf{V} \tau
$$

where we assume that $|\mathbf{X}|$ is small and that the $X$-direction was the normal to the transition zone when it crossed $\mathbf{x}$ at time $t-\tau$. A leading order estimate will now be made of $\mathrm{I}\left(\tau_{-}, \tau_{+}\right)$, using the same technique as in (3.35). We replace (3.14) by

$$
r=\frac{X}{\epsilon}
$$

and assume that $k_{s}, k_{l}$ do not vary significantly over $\left(\tau_{-}, \tau_{+}\right)$. Then, putting

$$
\mathbb{I}\left(\tau_{-}, \tau_{+}\right)=\mathbf{I}_{s}+\mathbf{I}_{l},
$$

where $\mathbf{I}_{s}, \mathbf{I}_{l}$ represent the first and second terms of (3.93) $)_{1}$, we have

$$
I_{s X} \approx \frac{k_{s}(\tau)}{\epsilon} \int_{\rho_{-}}^{\rho_{+}}\left[h_{0 s} u_{0 r}\right]\left(r-V_{x} \frac{\rho}{\epsilon}, Y-V_{y} \rho, Z-V_{z} \rho\right) \mathrm{d} \rho, \quad \tau+\rho_{ \pm}=\tau_{ \pm},
$$

for the $X$-component of $\mathbf{I}_{s}$. The $Y, Z$ components are negligible compared with $I_{s X}$. Then, corresponding to the leading term in (3.35) (without the overall differentiation with respect $r$ ), we have

$$
\begin{aligned}
I_{S X} & \approx k_{s}(\tau) \int_{\frac{\rho_{-}}{\epsilon}}^{\frac{\rho_{+}}{\epsilon}}\left[h_{s 0} u_{0 r}\right]\left(r-V_{x} \alpha, Y-V_{y} \epsilon \alpha, Z-V_{z} \epsilon \alpha\right) \mathrm{d} \alpha, \\
& \approx \frac{k_{s}(\tau)}{V_{x}} \int_{r_{-}}^{r_{+}} h_{0 s}\left(r^{\prime}\right) \frac{\partial}{\partial r^{\prime}} u_{0}\left(r^{\prime}, Y, Z\right) \mathrm{d} r^{\prime} \\
r^{\prime} & =r-V_{x} \alpha, \quad r_{ \pm}=r-V_{x} \frac{\rho_{ \pm}}{\epsilon} .
\end{aligned}
$$

To leading order, we can replace $r_{ \pm}$by $\pm \infty$. Giving a similar representation for $I_{I X}$, carrying out a partial integration and referring to (3.86), we find that

$$
\mathbf{I}\left(\tau_{-}, \tau_{+}\right)=\nabla \tau\left\{k_{s}(\tau) u_{0}(\infty, Y, Z)-k_{l}(\tau) u_{0}(-\infty, Y, Z)-\left(k_{s}(\tau)-k_{l}(\tau)\right) u_{a}(Y, Z)\right\},
$$

where $u_{a}(Y, Z)$ is defined by $(3.42)_{2}$ with $Y, Z$ which are small, replacing $y, z$. It is given by (3.56). Let us introduce the notation

$$
[u]_{ \pm}=u\left(\mathbf{x}-\mathbf{V} \tau_{-}\right) \pm u\left(\mathbf{x}-\mathbf{V} \tau_{+}\right) .
$$

The quantities $u( \pm \infty, Y, Z)$ have been replaced by $u\left(\mathbf{x}-\mathbf{V} \tau_{\mp}\right)$. In fact, by virtue of the steady-state assumption, [u$]_{-}$is the quantity $\left[\bar{u}_{0}\right]_{-}$ obeying (3.59), evaluated at the small quantities $Y, Z$ rather than at the small quantities $y, z$. We have

$$
\mathbf{I}\left(\tau_{-}, \tau_{+}\right)=\nabla \tau k_{J}(\tau)\left[u_{0}\right]_{-}, \quad k_{J}(\tau)=k_{s}(\tau)(1-J)+k_{l}(\tau) J,
$$

where $J$ is defined in (3.56) 5 . This result gives that $U=J$ in (3.92), since the expression in (3.92) is $\mathbf{V} \cdot \mathbf{I}\left(\tau_{-}, \tau_{+}\right)$.

Note that, using the notation of (3.88),

$$
\begin{aligned}
\nabla \int_{0}^{\infty} k(s) u(\mathbf{x}-\mathbf{V} s) \mathrm{d} s= & \nabla\left\{\int_{0}^{\tau_{-}(\mathbf{x})} k_{s}(s) u(\mathbf{x}-\mathbf{V} s) \mathrm{d} s+\int_{\tau_{+}(\mathbf{x})}^{\infty} k_{l}(s) u(\mathbf{x}-\mathbf{V} s) \mathrm{d} s\right\} \\
= & \int_{0}^{\tau_{-}(\mathbf{x})} k_{s}(s) \nabla u(\mathbf{x}-\mathbf{V} s) \mathrm{d} s+\int_{\tau_{+}(\mathbf{x})}^{\infty} k_{l}(s) \nabla u(\mathbf{x}-\mathbf{V} s) \mathrm{d} s \\
& +\nabla \tau\left(k_{s}(\tau) u\left(\mathbf{x}-\mathbf{V} \tau_{-}\right)-k_{l}(\tau) u\left(\mathbf{x}-\mathbf{V} \tau_{+}\right)\right) .
\end{aligned}
$$

Let us define

$$
k_{-}(\tau)=\frac{1}{2}\left(k_{s}(\tau)-k_{l}(\tau)\right)
$$


From (3.93) and (3.101),

$$
\begin{aligned}
\mathbf{q}(\mathbf{x}) & =-\int_{0}^{\tau_{-}(\mathbf{x})} k_{s}(s) \nabla u(\mathbf{x}-\mathbf{V} s) \mathrm{d} s-\int_{\tau_{+}(\mathbf{x})}^{\infty} k_{l}(s) \nabla u(\mathbf{x}-\mathbf{V} s) \mathrm{d} s-k_{J}(\tau) \nabla \tau[u]_{-} \\
& =-\nabla \int_{0}^{\infty} k(s) u(\mathbf{x}-\mathbf{V} s) \mathrm{d} s+\nabla \tau k_{-}(\tau)[u]_{J}, \\
{[u]_{J} } & =2\left(u\left(\mathbf{x}-\mathbf{V} \tau_{-}\right) J+u\left(\mathbf{x}-\mathbf{V} \tau_{+}\right)(1-J)\right) .
\end{aligned}
$$

The second form follows from (3.102) and (3.100).

Comparing (3.104) and (3.83) for $\tau(\mathbf{x})=0$, we see that the discontinuity of $\mathbf{q}(\mathbf{x})$ across $s$ is given by

$$
\mathbf{q}\left(\mathbf{x}^{+}\right)-\mathbf{q}\left(\mathbf{x}^{-}\right)=-k_{J}(0) \nabla \tau[u]_{-}=-\frac{V_{J}^{2} V_{x} \gamma}{V_{x}^{2}-V_{J}^{2}},
$$

by virtue of (3.59) and (3.86).

Observe that $u(\mathbf{X})$ is given by (3.10) with $\mathbf{X}$, defined by (3.94), replacing $\mathbf{X}$. Then

$$
\begin{aligned}
\frac{\partial}{\partial x_{i}} u(\mathbf{x}-\mathbf{V} \tau) & =\frac{\partial}{\partial x_{i}} u(\mathbf{X})=\sum_{j=1}^{3} \frac{\partial}{\partial X_{j}} u(\mathbf{X}) \frac{\partial X_{j}}{\partial x_{i}} \\
& =\frac{\partial}{\partial X_{i}} u(\mathbf{X})-\frac{\partial \tau}{\partial x_{i}} \sum_{j=1}^{3} V_{j} \frac{\partial}{\partial X_{j}} u(\mathbf{X}) .
\end{aligned}
$$

Using (3.10) and (3.86), we see that $\nabla u(\mathbf{x}-\mathbf{V} \tau)$ is negligible for $Y, Z$ close to zero.

Letting $\dot{u}=\mathbf{V} \cdot \nabla u=-\nabla \cdot \mathbf{q}(\mathbf{x})$ and using the first form of (3.104), we see that the heat equation becomes,

$$
\begin{aligned}
& \mathbf{V} \cdot \nabla u=\int_{0}^{\tau_{-}(\mathbf{x})} k_{s}(s) \Delta u(\mathbf{x}-\mathbf{V} s) \mathrm{d} s+\int_{\tau_{+}(\mathbf{x})}^{\infty} k_{l}(s) \Delta u(\mathbf{x}-\mathbf{V} s) \mathrm{d} s+j(\mathbf{x}), \\
& j(\mathbf{x})=\left(k_{J}^{\prime}(\tau)|\nabla \tau|^{2}+k_{J}(\tau) \Delta \tau\right)[u]_{-}+\nabla \tau \cdot\left(\left.k_{s}(\tau) \nabla u(\mathbf{x}-\mathbf{V} s)\right|_{s=\tau_{-}(\mathbf{x})}-\left.k_{l}(\tau) \nabla u(\mathbf{x}-\mathbf{V} s)\right|_{s=\tau_{+}(\mathbf{x})}\right)
\end{aligned}
$$

where a term containing $\nabla[u]_{-}$has been neglected by virtue of the property demonstrated from (3.106). Note that the gradients of $u$ in the last term are not negligible since $\tau_{ \pm}(\mathbf{x})$ in the arguments are not differentiated. ${ }^{4}$ Using the approximations (3.86) and (3.87), we have, in the notation of (3.76),

$$
j(\mathbf{x})=\frac{C_{J}(\tau)}{V_{x}^{2}}[u]_{-}+\frac{1}{V_{x}}\left(\left.k_{s}(\tau) u_{x}(\mathbf{x}-\mathbf{V} s)\right|_{s=\tau_{-}(\mathbf{x})}-\left.k_{l}(\tau) u_{x}(\mathbf{x}-\mathbf{V} s)\right|_{s=\tau_{+}(\mathbf{x})}\right)
$$

where, as noted earlier, $[u]_{-}$is equal to $\left[\bar{u}_{0}\right]_{-}$, given by (3.59). For $\tau=0$, the quantity $j(\mathbf{x})$ is given by $V_{x}\left[\bar{u}_{0 x}\right]_{-}$by virtue of (3.77).

For $s>\tau, u$ in (3.107) is the solution of (3.82). Relations (3.82) and (3.107) are thus an inter-related system of integro-partial differential equations, to be solved subject to boundary conditions for the body as a whole and (3.59) and (3.77) at the transition zone.

Using the second form of (3.104), we can write the solid phase heat equation in the form

$$
\begin{aligned}
\mathbf{V} \cdot \nabla u & =\Delta \int_{0}^{\infty} k(s) u(\mathbf{x}-\mathbf{V} s) \mathrm{d} s-\left(k_{-}^{\prime}(\tau)|\nabla \tau|^{2}+k_{-}(\tau) \Delta \tau\right)[u]_{J} \\
& =\Delta \int_{0}^{\infty} k(s) u(\mathbf{x}-\mathbf{V} s) \mathrm{d} s-\frac{C_{-}(\tau)}{V_{x}^{2}}[u]_{J} .
\end{aligned}
$$

\subsection{A simple example}

We consider the solution of the Eqs. (3.82) and (3.109) for the case where the body $\mathscr{B}$ occupies all of $\mathcal{R}^{3}$ and the surface $s$ is the $(y, z)$ plane, so that $\mathcal{K}_{0}=V_{y}=V_{z}=0$. These equations reduce to

$$
\begin{aligned}
V_{x}^{2} u_{x}(x) & =\frac{\mathrm{d}^{2}}{\mathrm{~d} x^{2}} \int_{-\infty}^{x} k_{l}\left(\frac{x-x^{\prime}}{V_{x}}\right) u\left(x^{\prime}\right) \mathrm{d} x^{\prime}, \quad x<0, \\
& =\frac{\mathrm{d}^{2}}{\mathrm{~d} x^{2}} \int_{-\infty}^{x} k\left(\frac{x-x^{\prime}}{V_{x}}\right) u\left(x^{\prime}\right) \mathrm{d} x^{\prime}-\frac{1}{V_{x}} k_{-}^{\prime}\left(\frac{x}{V_{x}}\right)[u]_{J}, \quad x>0,
\end{aligned}
$$

with a change of variable to $x^{\prime}=x-V_{x}$ s. In the second form, $k$ is defined by (3.88). The heat flux equations (3.83) and (3.104) become

$$
\begin{aligned}
q(x) & =-\frac{1}{V_{x}} \frac{\mathrm{d}}{\mathrm{d} x} \int_{-\infty}^{x} k_{l}\left(\frac{x-x^{\prime}}{V_{x}}\right) u\left(x^{\prime}\right) \mathrm{d} x^{\prime} \quad x<0 \\
& =-\frac{1}{V_{x}} \frac{\mathrm{d}}{\mathrm{d} x} \int_{-\infty}^{x} k\left(\frac{x-x^{\prime}}{V_{x}}\right) u\left(x^{\prime}\right) \mathrm{d} x^{\prime}+\frac{1}{V_{x}} k_{-}\left(\frac{x}{V_{x}}\right)[u]_{J}, \quad x>0 .
\end{aligned}
$$

\footnotetext{
4 This last term and the corresponding quantity in (4.60) were not included in [1].
} 
Let us take the simplest case, namely the Cattaneo model, where ${ }^{5}$

$$
\begin{aligned}
& k_{l}\left(\frac{x}{V_{x}}\right)=k_{l}(0) \mathrm{e}^{-\alpha x}=V_{l}^{2} \mathrm{e}^{-\alpha x}, \quad \alpha>0, \\
& k_{s}\left(\frac{x}{V_{x}}\right)=k_{s}(0) \mathrm{e}^{-\lambda x}=V_{s}^{2} \mathrm{e}^{-\lambda x}, \quad \lambda>0
\end{aligned}
$$

and

$$
k_{l}^{\prime}\left(\frac{x}{V_{x}}\right)=-\alpha V_{l}^{2} V_{x} \mathrm{e}^{-\alpha x}, \quad k_{s}^{\prime}\left(\frac{x}{V_{x}}\right)=-\lambda V_{s}^{2} V_{x} \mathrm{e}^{-\lambda x} .
$$

We assume, in this subsection that $V_{x}^{2}<\min \left(V_{l}^{2}, V_{s}^{2}\right)$. Consider the case where $x<0$. The solution is given by

$$
u(x)=a+A \mathrm{e}^{\beta x}, \quad u_{x}(x)=\beta A \mathrm{e}^{\beta x}, \quad \beta=\frac{\alpha V_{x}^{2}}{V_{l}^{2}-V_{x}^{2}}>0,
$$

where $a, A$ are constants.

For $x>0,(3.110)$ becomes

$$
V_{x}^{2} u_{x}(x)=V_{s}^{2} \frac{\mathrm{d}^{2}}{\mathrm{~d} x^{2}}\left\{\int_{0}^{x} \mathrm{e}^{-\lambda\left(x-x^{\prime}\right)} u\left(x^{\prime}\right) \mathrm{d} x^{\prime}\right\}+\alpha V_{l}^{2}\left(a+\frac{\alpha}{\alpha+\beta} A\right) \mathrm{e}^{-\alpha x}+\frac{1}{2}\left(\lambda V_{s}^{2} \mathrm{e}^{-\lambda x}-\alpha V_{l}^{2} \mathrm{e}^{-\alpha x}\right)[u]_{J},
$$

where the second term on the right follows from integration over $\mathcal{R}^{-}$of the solution (3.114). We seek a solution of the form

$$
u(x)=b+B \mathrm{e}^{-\alpha x}, \quad x>0,
$$

so that

$$
[u]_{J}=2(J(b+B)+(1-J)(a+A)) .
$$

Relation (3.115) becomes

$$
-\alpha V_{x}^{2} B \mathrm{e}^{-\alpha x}=V_{s}^{2}\left[-\lambda b \mathrm{e}^{-\lambda x}+\frac{B}{\lambda-\alpha}\left(\alpha^{2} \mathrm{e}^{-\alpha x}-\lambda^{2} \mathrm{e}^{-\lambda x}\right)+\frac{\lambda}{2}[u]_{J} \mathrm{e}^{-\lambda x}\right]+\alpha V_{l}^{2} \mathrm{e}^{-\alpha x}\left[a+\frac{\alpha}{\alpha+\beta} A-\frac{1}{2}[u]_{J}\right] .
$$

Comparing coefficients of the exponentials, we obtain

$$
\begin{aligned}
\lambda b=- & \frac{\lambda^{2} B}{\lambda-\alpha}+\frac{\lambda}{2}[u]_{J}, \\
-\alpha V_{x}^{2} B & =\frac{\alpha^{2} V_{s}^{2} B}{\lambda-\alpha}+\alpha V_{l}^{2}\left[a+\frac{\alpha}{\alpha+\beta} A-\frac{1}{2}[u]_{J}\right] \\
& =\frac{\alpha^{2} V_{s}^{2} B}{\lambda-\alpha}+\alpha V_{l}^{2}(a+A)-\alpha V_{x}^{2} A-V_{l}^{2} \frac{\alpha}{2}[u]_{J}
\end{aligned}
$$

where (3.114) ${ }_{3}$ has been used. Relation (3.73) becomes, in this case, with the aid of (3.21), (3.113), (3.114) and (3.116),

$$
\left(V_{x}^{2}-V_{s}^{2}\right)(-\alpha B)-\left(V_{x}^{2}-V_{l}^{2}\right)(\beta A)=-\lambda V_{s}^{2}(b+B)+\alpha V_{l}^{2}(a+A)+\left(\lambda V_{s}^{2}-\alpha V_{l}^{2}\right) u_{a}
$$

or, using $(3.114)_{3}$ in the second term on the left,

$$
\alpha V_{x}^{2}(A-B)=-\lambda V_{s}^{2} b-(\alpha+\lambda) V_{s}^{2} B+\alpha V_{l}^{2}(a+A)+\left(\lambda V_{s}^{2}-\alpha V_{l}^{2}\right) u_{a}
$$

Consider the first term on the right of $(3.119)_{3}$. Using the identity

$$
\frac{\alpha^{2}}{\lambda-\alpha}=\frac{\lambda^{2}}{\lambda-\alpha}-(\lambda+\alpha)
$$

and (3.119) $)_{1}$, we can write this in the form

$$
\frac{\alpha^{2} V_{s}^{2} B}{\lambda-\alpha}=-\lambda V_{s}^{2}\left(b-\frac{1}{2}[u]_{J}\right)-(\lambda+\alpha) V_{s}^{2} B .
$$

Substituting this into $(3.119)_{3}$, comparing with (3.121) and observing that

$$
2 u_{a}=[u]_{J},
$$

by virtue of (3.56) and (3.104) 3 we see that they are identical. Thus, this model provides a relation in agreement with (3.73), which generalizes a result in [1].

5 The quantities $\alpha, \beta$ are not related to those in (3.3). 
Reorganizing (3.121) with the aid of (3.124) and (3.117), we obtain

$$
\alpha V_{x}^{2}(A-B)=-\left(\lambda V_{s}^{2}(1-J)+\alpha V_{l}^{2} J\right)[u]_{-}-\alpha V_{s}^{2} B .
$$

Relation (3.119) 1 yields, after some manipulation, the alternative forms

$$
A=b-a+\frac{B(\lambda(1-J)+\alpha J)}{(\lambda-\alpha)(1-J)}
$$

or

$$
[u]_{-}=\frac{B \alpha}{(\alpha-\lambda)(1-J)} .
$$

Substituting from (3.127) into (3.125), we can solve (3.125) and (3.126) to give

$$
\begin{aligned}
B & =\frac{(b-a)(\alpha-\lambda) V_{x}^{2}(1-J)}{\alpha\left(V_{x}^{2}-V_{J}^{2}\right)} \\
A & =B-\frac{(b-a) V_{J}^{2}}{V_{x}^{2}-V_{J}^{2}} \\
& =\frac{(b-a)}{\alpha}\left[\frac{(\alpha-\lambda) V_{x}^{2}(1-J)-\alpha V_{J}^{2}}{\left(V_{x}^{2}-V_{J}^{2}\right)}\right] \\
& =(b-a)\left[\frac{W V_{x}^{2}-V_{J}^{2}}{\left(V_{x}^{2}-V_{J}^{2}\right)}\right], \quad W=\left(1-\frac{\lambda}{\alpha}\right)(1-J) .
\end{aligned}
$$

It would perhaps be reasonable to assume that the liquid phase has a memory no longer than the solid phase so that $\alpha \geq \lambda, A>0$ and $B \leq 0$.

If $a=u(-\infty), b=u(\infty)$ are regarded as input data, then these relations for $A, B$ represent a solution to the problem. Note that it follows from (3.127) and (3.128) that

$$
[u]_{-}=\frac{(b-a) V_{x}^{2}}{V_{x}^{2}-V_{J}^{2}} .
$$

Comparing (3.129) with (3.59), we see that

$$
\gamma(\mathbf{V})=b-a,
$$

so that in fact $b$ and $a$ cannot be chosen independently. They must obey this relation in order to give rise to a steady-state configuration with transition zone velocity $-\mathbf{V}$.

The implication of (3.129) that $b>a$ was regarded as unphysical in [1]; it is unquestionably surprising. However, given the viewpoint adopted here that $a, b$ are input data, it can be rationalized as follows. We create a boundary temperature field such that $u(\infty)=b$. It is reasonable to assume that $b<0$. Then, in order to drive a solidification process at velocity $-\mathbf{V}$, we must create a boundary temperature $u(-\infty)=a$, where this is below the solidification temperature of the liquid. The temperature increases to $a+A$ at $x=0^{-}$, which must be greater that $b+B$ at $x=0^{+}$since (3.129) gives that $[u]_{-}$is negative. However, the region of the liquid for which $x<\log (|a / A|) / \beta$ will be either solidified or super-cooled. This is based on the assumption that $a+A>0$. If not, then the entire liquid is at a temperature less that the solidification temperature.

The heat flux (3.111) has the form

$$
q(x)=\left\{\begin{array}{l}
-V_{x} B \mathrm{e}^{-\alpha x}, \quad x>0 \\
-V_{x} A \mathrm{e}^{\beta x}, \quad x<0
\end{array}\right.
$$

which can be shown using (3.114) and (3.119). Taking $A>0, B<0$, we have that the heat flux for $x>0$ is in a positive direction and, for the liquid phase, the flow is in a negative direction. The discontinuity across the transition zone is given by

$$
-V_{x}(B-A)=\frac{\gamma V_{J}^{2} V_{x}}{V_{J}^{2}-V_{x}^{2}}
$$

in agreement with (3.105). The Fourier limit of all these formulae is obtained as outlined after (2.23). In this limit, the right-hand side of (3.132) reduces to $\gamma V_{x}$. The exact result is greater than $\gamma V_{x}$ so that memory effects create dissipation, which gives off extra heat in addition to the latent heat.

\section{General asymptotic analysis}

We now carry out a more formal asymptotic analysis of these equations, relying substantially on $[7,6,3,8]$. The following notation is introduced:

$$
\begin{aligned}
& k_{i} \circ n^{t}\left(\mathbf{x}, s_{1}, s_{2}\right)=\int_{s_{1}}^{s_{2}} k_{i}(s) n^{t}(\mathbf{x}, s) \mathrm{d} s, \quad k_{i} \circ n^{t}(\mathbf{x})=k_{i} \circ n^{t}(\mathbf{x}, 0, \infty) \\
& k_{i} \circ n^{t}\left(\mathbf{x}, s_{2}\right)=k_{i} \circ n^{t}\left(\mathbf{x}, 0, s_{2}\right), \quad i=l, s
\end{aligned}
$$

where $\mathbf{x}$ is often omitted from the arguments. 
Let us assume that the region $\mathscr{B}_{\alpha}$ occupied by the solid phase $\alpha$ is expanding and that occupied by the liquid phase $\mathscr{B}_{\beta}$ is contracting or ablating. Thus, the transition zone is moving into the liquid phase. We shall use the notation $\mathscr{B}_{\alpha}, \mathscr{B}_{\beta}$ to include the respective portions of $\mathcal{B}$ on either side of $\Sigma(t)$.

Let $d(\mathbf{x}, t)$ be the signed distance between $\mathbf{x}$ and $\Sigma(t)$ where, at $\mathbf{x}$ outside of $\mathcal{Z}$,

$$
\begin{aligned}
d(\mathbf{x}, t)<0 & \text { if } \varphi(\mathbf{x}, t, \epsilon)=0 \text { (liquid) } \\
>0 & \text { if } \varphi(\mathbf{x}, t, \epsilon)=1 \text { (solid). }
\end{aligned}
$$

Let

$$
r(\mathbf{x}, t)=\frac{1}{\epsilon} d(\mathbf{x}, t) .
$$

We now carry out an outer expansion of $\varphi, u$ and other variables in the regions outside of the transition zone; and an inner expansion about $\Sigma(t)$. Let $(d(\mathbf{x}, t), \zeta(\mathbf{x}, t))$ with $d$ perpendicular and $\zeta=\left(\zeta_{1}, \zeta_{2}\right) \in \mathcal{R}^{2}$ tangential to $\Sigma(t)$, be a local orthogonal coordinate system on this surface. Let $\chi$ be any field of interest. The outer expansion is given by

$$
\bar{\chi}(\mathbf{x}, t, \epsilon)=\bar{\chi}_{0}(d(\mathbf{x}, t), \zeta(\mathbf{x}, t), t)+\epsilon \bar{\chi}_{1}(d(\mathbf{x}, t), \zeta(\mathbf{x}, t), t)+O\left(\epsilon^{2}\right)
$$

and the inner expansion,

$$
\chi(\mathbf{x}, t, \epsilon)=\chi_{0}(r(\mathbf{x}, t), \zeta(\mathbf{x}, t), t)+\epsilon \chi_{1}(r(\mathbf{x}, t), \zeta(\mathbf{x}, t), t)+O\left(\epsilon^{2}\right)
$$

where we note the use of the stretched variable $r$. The matching condition is

$$
\chi(d / \epsilon, \zeta, t, \epsilon)=\chi(r, \zeta, t, \epsilon)=\bar{\chi}(\epsilon r, \zeta, t, \epsilon)
$$

for large $r$ and $\epsilon r$ small. Specifically, this relation will hold when $(\epsilon r, \zeta)$ is in the vicinity of $\Sigma_{0}$ or $\Sigma_{1}$, defined by (2.26), at time $t$. Differentiating, we obtain

$$
\chi_{r}(r, \zeta, t, \epsilon)=\epsilon \bar{\chi}_{d}(\epsilon r, \zeta, t, \epsilon), \quad \chi_{r}(r, \zeta, t, \epsilon)=\epsilon^{2} \bar{\chi}_{d d}(\epsilon r, \zeta, t, \epsilon)
$$

and so on. Relations (4.6) and (4.7) give

$$
\begin{aligned}
& \chi_{0}( \pm \infty, \zeta, t)=\bar{\chi}_{0}(0 \pm, \zeta, t), \quad \chi_{1 r}( \pm \infty, \zeta, t)=\bar{\chi}_{0 d}(0 \pm, \zeta, t,) \\
& \chi_{0 r}( \pm \infty, \zeta, t)=\chi_{0 r r}( \pm \infty, \zeta, t)=0
\end{aligned}
$$

and

$$
\chi_{0 r}(r, \zeta, t)=O(\epsilon), \quad \chi_{0 r r}(r, \zeta, t)=O\left(\epsilon^{2}\right)
$$

where $(\epsilon r, \zeta)$ is in the vicinity of $\Sigma_{0}$ or $\Sigma_{1}$ at time $t$.

For $\varphi$ in particular, we have the equivalent of (3.18) and (3.19) or, for $\epsilon=0$,

$$
\bar{\varphi}_{0}(d, \zeta, t)= \begin{cases}1, & d>0 \\ 0, & d<0\end{cases}
$$

for $\bar{\varphi}_{0}$ so that

$$
\bar{\varphi}_{i}(d, \zeta, t)=0, \quad i=1,2 \ldots
$$

\subsection{Transition zone relations}

Let us now consider the inner expansions. We have

$$
\nabla \chi(r, \zeta, t, \epsilon)=\chi_{d}(r, \zeta, t, \epsilon) \nabla d+\sum_{i=1}^{2} \chi_{\zeta_{i}}(r, \zeta, t, \epsilon) \nabla \zeta_{i}, \quad r=\frac{d}{\epsilon}
$$

and

$$
\Delta \chi(r, \zeta, t, \epsilon)=\chi_{d d}(r, \zeta, t, \epsilon)|\nabla d|^{2}+\chi_{d}(r, \zeta, t, \epsilon) \Delta d+\sum_{i=1}^{2} \chi_{\zeta_{i}}(r, \zeta, t, \epsilon) \Delta \zeta_{i}+\sum_{i=1}^{2} \chi_{\zeta_{i} \zeta_{i}}(r, \zeta, t, \epsilon)\left|\nabla \zeta_{i}\right|^{2}
$$

where the orthogonality of the coordinate system $(d, \zeta)$ has been used. Also

$$
\dot{\chi}(r, \zeta, t, \epsilon)=\chi_{d}(r, \zeta, t, \epsilon) \dot{d}+\sum_{i=1}^{2} \chi_{\zeta_{i}}(r, \zeta, t, \epsilon) \dot{\zeta}_{i}+\chi_{t}(r, \zeta, t, \epsilon)
$$

where $\chi_{t}$ is the derivative with respect to the explicit occurrence of $t$. We also have

$$
\nabla \cdot(h \nabla \chi)=\nabla h \cdot \nabla \chi+h \Delta \chi .
$$


Let $\mathbf{x} \in \mathcal{Z}$ be such that $\left|\mathbf{x}-\mathbf{x}_{0}\right|$ is $O(\epsilon)$, where $\mathbf{x}_{0} \in \Sigma(t)$. Then, for any slowly varying function $f$ in the transition zone (i.e. not $\varphi$ or $u$ or their derivatives)

$$
f(\mathbf{x}, t)=f\left(\mathbf{x}_{0}, t\right)+O(\epsilon) .
$$

Thus, we have

$$
\begin{aligned}
& \nabla d(\mathbf{x}, t)=\nabla d\left(\mathbf{x}_{0}, t\right)+O(\epsilon)=\mathbf{m}_{0}\left(\zeta\left(\mathbf{x}_{0}, t\right), t\right)+O(\epsilon) \\
& \Delta d(\mathbf{x}, t)=\Delta d\left(\mathbf{x}_{0}, t\right)+O(\epsilon)=-\mathcal{K}_{0}\left(\zeta\left(\mathbf{x}_{0}, t\right), t\right)+O(\epsilon) \\
& \dot{d}(\mathbf{x}, t)=\dot{d}\left(\mathbf{x}_{0}, t\right)+O(\epsilon)=V_{0}\left(\zeta\left(\mathbf{x}_{0}, t\right), t\right)+O(\epsilon)
\end{aligned}
$$

where $\mathbf{m}_{0}(\zeta, t), V_{0}(\zeta, t)$ and $\mathcal{K}_{0}(\zeta, t)$ denote respectively the unit normal to $\Sigma(t)$, the normal velocity of $\Sigma(t)$, directed into the liquid, and twice its mean curvature at $\mathbf{x}_{0}$. The quantity $\mathcal{K}_{0}(\zeta, t)$ is positive if the solid phase is convex at the point under consideration. We choose the sign convention of $(4.17)_{3}$ so that $V_{0}$ corresponds to $V_{x}$ in (3.1) and (3.2).

Note that, from (4.3),

$$
\chi_{d}=\frac{1}{\epsilon} \chi_{r} .
$$

Field quantities $\chi_{r}$ are $O(1)$ while $\chi_{d}$ are $O(1 / \epsilon)$.

To leading order, (4.12) becomes

$$
\nabla \chi_{0}(r, \zeta, t)=\chi_{0 d}(r, \zeta, t) \mathbf{m}_{0}
$$

and (4.13) gives

$$
\Delta \chi_{0}(r, \zeta, t)=\chi_{\text {odd }}(r, \zeta, t)-\chi_{\text {od }}(r, \zeta, t) \mathcal{K}_{0}(\zeta, t) .
$$

Also, (4.14) becomes

$$
\dot{\chi}_{0}(r, \zeta, t)=\chi_{0 d}(r, \zeta, t) V_{0}(\zeta, t)=\frac{1}{\epsilon} \chi_{0 r}(r, \zeta, t) V_{0}(\zeta, t) .
$$

The tangential contributions are $O(1)$ and can be neglected. For $\mathbf{x} \in \mathcal{Z}$, (cf. (3.11))

$$
k_{i} \circ h_{i} \nabla u^{t}(\mathbf{x})=k_{i} \circ h_{i} \nabla u^{t}\left(\mathbf{x}, s_{-}\right)+k_{i} \circ h_{i} \nabla u^{t}\left(\mathbf{x}, s_{-}, \infty\right), \quad i=l, s,
$$

where the dimensionless time parameter $s_{-}(\mathbf{x}, t, \epsilon)$ is the portion of the time integration for which $\mathbf{x} \in \mathcal{Z}$. Thus, in the first term on the right of (4.22), $\mathbf{x} \in \mathcal{Z}$ while in the second, $\mathbf{x} \in \mathscr{B} \backslash \mathcal{Z}$, specifically in the liquid phase. For the first term on the right of (4.22), $r$ is used in $\nabla u^{t}\left(\mathbf{x}, s_{-}\right)$while $d$ is used in the second term. For $i=s$, the second term on the right is zero. We have $\mathbf{x} \in \Sigma_{0}$ at time $t-s_{-}$. Referring to the comment after (3.13), we see that the dimensionless quantity $s_{-}$is $O(\epsilon)$. We neglect the $\epsilon$ dependence of $s_{-}(\mathbf{x}, t, \epsilon)$ and justify this later.

The dependence of field quantities on space coordinates is often omitted. However, the indicated time dependence, if $(d, \zeta)$ or $(r, \zeta)$ are omitted, is the complete time dependence, including that which occurs through $(d, \zeta)$.

\subsection{The order parameter relation}

Let us write $(2.19)_{1}$, keeping only those terms with $O(\epsilon)$ contributions:

$$
\varphi_{\pi}-f^{\prime}(\varphi)-u \beta \epsilon g^{\prime}(\varphi)-\epsilon \alpha V_{0} \varphi_{r}-\epsilon \varphi_{r} \mathcal{K}_{0}+O\left(\epsilon^{2}\right)=0 .
$$

Relations (4.18), (4.20) and (4.21) have been used. All quantities are understood to be evaluated at time $t$.

To leading order, $(4.23)_{1}$ becomes (cf. (3.27))

$$
\varphi_{0 \pi r}-f^{\prime}\left(\varphi_{0}\right)=0 .
$$

The quantity $\varphi_{0}$ is given by the solution of (4.24), subject to the boundary conditions given by the $O(1)$ matching relations $(4.8)_{1}$ :

$$
\varphi_{0}(-\infty, \zeta, t)=0, \quad \varphi_{0}(+\infty, \zeta, t)=1
$$

and has the explicit form given by (3.29) depending only on $r$. This solution obeys

$$
\varphi_{0}(0, \zeta, t)=\frac{1}{2}
$$

giving that $r=0$ defines $\Sigma(t)$, consistent with the choice made after (2.26).

The $O(\epsilon)$ term of (4.23) 1 yields ( $c f .(3.30))$

$$
\varphi_{1 r r}-f^{\prime \prime}\left(\varphi_{0}\right) \varphi_{1}-u_{0} \beta g^{\prime}\left(\varphi_{0}\right)-\alpha V_{0} \varphi_{0 r}-\mathcal{K}_{0} \varphi_{0 r}=0,
$$

which leads to a relation of the form (3.32) with $V_{0}$ replacing $V_{x}$. 


\subsection{The heat equation}

The $O(\epsilon)$ contribution of the heat equation is now considered. Let us write $(2.19)_{2}$, keeping only those terms with $O(\epsilon)$ contributions:

$$
\sum_{i=l, s} k_{i} \circ\left(h_{i 0}^{t} u_{0 r}^{t}\right)_{r}\left(s_{-}\right)+\epsilon\left[-u_{0 r} V_{0}+\gamma g^{\prime}\left(\varphi_{0}\right) \varphi_{0 r} V_{0}\right]+O\left(\epsilon^{2}\right)=0 .
$$

We have multiplied (2.19) $)_{2}$ by $\epsilon^{2}$ in writing this equation. Relations (4.18), (4.20) and (4.21) have been used. The $h_{i 0}^{t}$ are functions of $\varphi_{0}^{t}(s)=\varphi_{0}^{t}(r(\mathbf{x}, t-s))$. Quantities that are not part of the convolution integrals are understood to be evaluated at time $t$. We have

$$
\begin{aligned}
k_{i} \circ\left(h_{i 0}^{t} u_{0 r}^{t}\right)_{r}\left(s_{-}\right) & =\int_{0}^{s_{-}} k_{i}(s)\left(h_{i 0}^{t} u_{0 r}^{t}\right)_{r}(s) \mathrm{d} s \\
& =\int_{t-s_{-}}^{t} k_{i}(t-s)\left(h_{i 0} u_{0 r}\right)_{r}(s) \mathrm{d} s, \quad i=l, s .
\end{aligned}
$$

It is assumed as before that $k_{i}(s), i=l, s$ does not vary greatly over the period from $s=0$ to $s_{-}$. Let us put

$$
v_{i}=h_{i} u_{r}, \quad i=l, s
$$

giving

$$
v_{i 0}=h_{i 0} u_{0 r}, \quad v_{i 1}=h_{i 0} u_{1 r}+h_{i 1} u_{0 r} \quad i=l, s .
$$

Now, from (4.21) and (4.18), we have

$$
\dot{v}_{i 0}(s)=\frac{V_{0}(t)}{\epsilon} v_{i 0 r}(s), \quad s \in\left[t-s_{-}, t\right], i=l, s,
$$

since

$$
V_{0}(s)=V_{0}(t)+(s-t) \dot{V}_{0}(t)=V_{0}(t)+O(\epsilon)
$$

because $\dot{V}_{0}(\zeta, t)$ is independent of $r$ and therefore of $O(1)$ by (4.14). Using (4.32), we see that

$$
\begin{aligned}
\int_{t-s_{-}}^{t} k_{i}(t-s) v_{i 0 r}(s) \mathrm{d} s & =\frac{\epsilon}{V_{0}(t)} \int_{t-s_{-}}^{t} k_{i}(t-s) \dot{v}_{i 0}(s) \mathrm{d} s \\
& =\frac{\epsilon}{V_{0}(t)}\left[k_{i}(0) v_{i 0}(t)-k_{i}\left(s_{-}\right) v_{i 0}\left(t-s_{-}\right)\right]+\frac{\epsilon}{V_{0}(t)} \int_{t-s_{-}}^{t} k_{i}^{\prime}(t-s) v_{i 0}(s) \mathrm{d} s \\
& =\frac{\epsilon}{V_{0}(t)} k_{i}(0)\left[v_{i 0}(t)-v_{i 0}\left(t-s_{-}\right)\right]-\frac{\epsilon}{V_{0}(t)} k_{i}^{\prime}(0) s_{-} v_{i 0}\left(t-s_{-}\right)+\frac{\epsilon k_{i}^{\prime}(0)}{V_{0}(t)} \int_{t-s_{-}}^{t} v_{i 0}(s) \mathrm{d} s, \quad i=l, s .
\end{aligned}
$$

From (4.9) $)_{1}$, it follows that, since $(r, \zeta)$ is on $\Sigma_{0}$ at time $t-s_{-}$then $v_{i 0}\left(r, \zeta, t-s_{-}\right)$is $O(\epsilon)$ and can be neglected, while $v_{i 0}(t)$ is $O(1)$. Thus, the first term is $O(\epsilon)$ and others, explicitly shown, are higher order, since

$$
\int_{t-s_{-}}^{t} v_{i 0}(s) \mathrm{d} s=O\left(s_{-}\right)=O(\epsilon)
$$

so that the last integral of (4.34) is $O\left(\epsilon^{2}\right)$. The previous term is $O\left(\epsilon^{3}\right)$ because of the factor $s_{-}$. Thus, the $O(\epsilon)$ terms of (4.28) give

$$
\left(k_{l}(0) h_{l 0}(t)+k_{s}(0) h_{s 0}(t)\right) u_{0 r}(t)-u_{0 r}(t) V_{0}^{2}(t)+\gamma g^{\prime}\left(\varphi_{0}(t)\right) \varphi_{0 r}(t) V_{0}^{2}(t)=0 .
$$

Integrating with respect to the variable $r$ over $R$ and using $(4.8)_{1}$ gives

$$
\begin{aligned}
& \left(V_{0}^{2}-V_{s}^{2}\right) u(\infty, \zeta, t)-\left(V_{0}^{2}-V_{l}^{2}\right) u(-\infty, \zeta, t)+\left(V_{s}^{2}-V_{l}^{2}\right) u_{a}=V_{0}^{2} \gamma \\
& u_{a}(\zeta, t)=\int_{-\infty}^{\infty} h_{s 0 r}\left(\varphi_{0}\left(r^{\prime}\right)\right) u_{0}\left(r^{\prime}, \zeta, t\right) \mathrm{d} r^{\prime}
\end{aligned}
$$

We adopt the notation corresponding to (3.45):

$$
\left[u_{0}\right]_{ \pm}=u_{0}(\infty, \zeta, t) \pm u_{0}(-\infty, \zeta, t)=\bar{u}_{0}(0+, \zeta, t) \pm \bar{u}_{0}(0-, \zeta, t)=\left[\bar{u}_{0}\right]_{ \pm} .
$$

The discussion from (3.46) to (3.64) also applies here, with $V_{0}$ replacing $V_{x}$. The quantity $u_{a}$ in (4.37) is given by (3.56) with $u_{0}( \pm \infty)=$ $u_{0}( \pm \infty, y, z)$ replaced by $u_{0}( \pm \infty, \zeta, t)$. Relation $(4.37){ }_{1}$ can be written as

$$
\left[u_{0}\right]_{-}=\left[\bar{u}_{0}\right]_{-}=\frac{V_{0}^{2} \gamma}{V_{0}^{2}-V_{J}^{2}}, \quad V_{0}=V_{0}(\zeta, t)
$$

which is equivalent to (3.59) in this more general context. Again, we must have $\gamma=\gamma\left(\mathbf{V}_{0}\right)$ or at least $\gamma=\gamma\left(V_{0}\right)$ where $\mathbf{V}_{0}(\zeta, t)=$ $\left(V_{0}(\zeta, t), V_{01}(\zeta, t), V_{02}(\zeta, t)\right)$, the quantities $V_{01}, V_{02}$ being the velocities in the directions $\zeta_{1}, \zeta_{2}$ on $\Sigma(t)$. This is because $\gamma$ must go to zero at least logarithmically in $\left(V_{0}^{2}-V_{l}^{2}\right),\left(V_{0}^{2}-V_{s}^{2}\right)$ as $V_{0} \rightarrow V_{l}, V_{s}$ respectively, to avoid a divergence in [ $\left.\bar{u}_{0}\right]$, as observed after (3.63). 
We now consider $O\left(\epsilon^{2}\right)$ terms. Let us apply (4.15) to the product $h_{i} \nabla u, i=l$, s. From (4.12), (4.13), (4.17) we have

$$
\begin{aligned}
\epsilon^{2} \int_{t-s_{-}}^{t} k_{i}(t-s) \nabla h_{i}(s) \cdot \nabla u(s) \mathrm{d} s & =\int_{t-s_{-}}^{t} k_{i}(t-s)\left[h_{i r}(s) u_{r}(s)|\nabla d|^{2}+\epsilon^{2} \sum_{j=1}^{2} h_{i \zeta_{j}} u_{\zeta_{j}}(s)\left|\nabla \zeta_{j}\right|^{2}\right] \mathrm{d} s, \\
\epsilon^{2} \int_{t-s_{-}}^{t} k_{i}(t-s) h_{i}(s) \Delta u(s) \mathrm{d} s= & \int_{t-s_{-}}^{t} k_{i}(t-s) h_{i}(s)\left[u_{r r}(s)|\nabla d|^{2}\right. \\
& \left.+\epsilon u_{r}(s) \Delta d+\epsilon^{2} \sum_{j=1}^{2} u_{\zeta_{j}}(s) \Delta \zeta_{j}+\epsilon^{2} \sum_{j=1}^{2} u_{\zeta i \zeta_{j}}(s)\left|\nabla \zeta_{j}\right|^{2}\right] \mathrm{d} s, \quad i=l, s .
\end{aligned}
$$

Because of the integration over $\left(t-s_{-}, t\right)$, the tangential terms are $O\left(\epsilon^{3}\right)$ and may be neglected. We write

$$
\begin{aligned}
& |\nabla d|^{2}=\left|\mathbf{m}_{0}+\epsilon \nabla d_{1}\right|^{2}=1+2 \epsilon \mathbf{m}_{0} \cdot \nabla d_{1}+O\left(\epsilon^{2}\right) \\
& \nabla d_{1}(\mathbf{x}, t)=\frac{1}{\epsilon}\left(\nabla d(\mathbf{x}, t)-\mathbf{m}_{0}(\zeta, t)\right)=O(1),
\end{aligned}
$$

giving

$$
\begin{aligned}
\epsilon^{2} \int_{t-s_{-}}^{t} k_{i}(t-s) \nabla h_{i}(s) \cdot \nabla u(s) \mathrm{d} s= & \int_{t-s_{-}}^{t} k_{i}(t-s)\left[h_{i 0 r}(s) u_{0 r}(s)+\epsilon\left(h_{i 0 r}(s) u_{1 r}(s)+h_{i 1 r}(s) u_{0 r}(s)\right.\right. \\
& \left.\left.+2 h_{i 0 r}(s) u_{0 r}(s) \mathbf{m}_{0} \cdot \nabla d_{1}\right)+O\left(\epsilon^{2}\right)\right] \mathrm{d} s, \\
\epsilon^{2} \int_{t-s_{-}}^{t} k_{i}(t-s) h_{i}(s) \Delta u_{0}(s) \mathrm{d} s= & \int_{t-s_{-}}^{t} k_{i}(t-s)\left[h_{i 0}(s) u_{0 r r}(s)+\epsilon\left(h_{i 0}(s) u_{1 r r}(s)+h_{i 1}(s) u_{0 r r}(s)\right.\right. \\
& \left.\left.+2 h_{i 0}(s) u_{0 r r}(s) \mathbf{m}_{0}(s) \cdot \nabla d_{1}(s)-\mathcal{K}_{0}(s) h_{i 0}(s) u_{0 r}(s)\right)+O\left(\epsilon^{2}\right)\right] \mathrm{d} s, \quad i=l, s .
\end{aligned}
$$

The effect of a small correction $s_{-} \rightarrow s_{-}+\epsilon \eta, \eta=O(\epsilon)$, in the integrals on the left would be the integrand evaluated at $t-s_{-}$ multiplied by $\eta \epsilon$, which is negligible to $O\left(\epsilon^{2}\right)$ (see (4.9)).

Using (4.14) and (4.18), we write

$$
\begin{aligned}
& \dot{u}(r, \zeta, t, \epsilon)=\frac{\dot{d}}{\epsilon} u_{r}(r, \zeta, t, \epsilon)+\sum_{i=1}^{2} u_{\zeta_{i}}(r, \zeta, t, \epsilon) \dot{\zeta}_{i}+u_{t}(r, \zeta, t, \epsilon) \\
& \dot{v}_{i}(r, \zeta, t, \epsilon)=\frac{\dot{d}}{\epsilon} v_{i r}(r, \zeta, t, \epsilon)+\sum_{j=1}^{2} v_{i \zeta_{j}}(r, \zeta, t, \epsilon) \dot{\zeta}_{j}+v_{i t}(r, \zeta, t, \epsilon), \quad i=l, s .
\end{aligned}
$$

Noting that, from (4.30) and $(4.31)_{2}$, we have

$$
\begin{aligned}
& \dot{u}_{1}(r, \zeta, t)=\frac{V_{0}(t)}{\epsilon} u_{1 r}(r, \zeta, t)+\frac{\delta(t)}{\epsilon} u_{0 r}(r, \zeta, t)+\frac{1}{\epsilon} \sum_{i=1}^{2} u_{0 \zeta_{i}}(r, \zeta, t) V_{0 i}+\frac{1}{\epsilon} u_{0 t}(r, \zeta, t) \\
& \dot{v}_{i 1}(r, \zeta, t)=\frac{V_{0}(t)}{\epsilon} v_{i 1 r}(r, \zeta, t)+\frac{\delta(t)}{\epsilon} v_{i 0 r}(r, \zeta, t)+\frac{1}{\epsilon} \sum_{j=1}^{2} v_{i 0 \zeta_{j}}(r, \zeta, t) V_{0 j}+\frac{1}{\epsilon} v_{i 0 t}(r, \zeta, t) \\
& \delta(t)=\frac{1}{\epsilon}\left(\dot{d}(\mathbf{x}, t)-V_{0}(\zeta, t)\right)=O(1), \quad V_{0 j}=\dot{\zeta}_{j} \quad j=1,2,
\end{aligned}
$$

where (4.17) $)_{3}$ has been used. The quantities $V_{0 i}(\zeta, t), i=1,2$ were introduced after (4.39). We have neglected the variation with $\mathbf{x}$ in the vicinity of $\mathbf{x}_{0}$ of $\delta(\mathbf{x}, t), \dot{\zeta}_{1}(\mathbf{x}, t), \dot{\zeta}_{2}(\mathbf{x}, t)$, by virtue of (4.16).

The $O\left(\epsilon^{2}\right)$ contribution to relation $(2.19)_{2}$ multiplied by $\epsilon^{2}$, or the $O(1)$ terms, without this factor $\epsilon^{2}$, has the form

$$
\begin{aligned}
u_{1 r} V_{0}+u_{0 r} \delta+\sum_{i=1}^{2} u_{0 \zeta_{i}} V_{0 i}+u_{0 t}= & \gamma[\dot{g}(\varphi)]_{2}+\sum_{i=l, s}\left\{-\frac{k_{i}(0)}{\epsilon V_{0}(t)} v_{i 0}\left(t-s_{-}\right)+\frac{k_{i}^{\prime}(0)}{V_{0}(t) \epsilon} \int_{t-s_{-}}^{t} v_{i 0}(s) \mathrm{d} s\right. \\
& +\frac{k_{i}(0)}{\epsilon} \int_{t-s_{-}}^{t}\left[v_{i 1 r}(s)+\left(\frac{1}{V_{0}(s)}-\frac{1}{V_{0}(t)}\right) \dot{v}_{i 0}(s)\right] \mathrm{d} s+\frac{2 k_{i}(0)}{\epsilon} \mathrm{m}_{0}(t) \cdot \nabla d_{1}(t) \int_{t-s_{-}}^{t} v_{i 0 r}(s) \mathrm{d} s \\
& \left.-\frac{\mathcal{K}_{0}(t) k_{i}(0)}{\epsilon} \int_{t-s_{-}}^{t} v_{i 0}(s) \mathrm{d} s\right\}+\int_{s_{-}}^{\infty} k_{l}(s) \Delta \bar{u}_{0}^{t}(s) \mathrm{d} s
\end{aligned}
$$

where (4.31), (4.42) and (4.44) $)_{1}$ have been used and $[\dot{g}(\varphi)]_{2}$ is the $O\left(\epsilon^{2}\right)$ term in $\epsilon^{2} \dot{g}(\varphi)$. The two terms on the second line are from (4.34) 2 , neglected as higher order in that context. A third term from (4.34) has been neglected as $O(\epsilon)$ in the context of (4.45). The second term on the third line is the $O\left(\epsilon^{2}\right)$ correction arising from the use of (4.33) on the right of (4.34) . To lowest order, this becomes

$$
\begin{aligned}
\int_{t-s_{-}}^{t}\left(\frac{1}{V_{0}(s)}-\frac{1}{V_{0}(t)}\right) \dot{v}_{i 0}(s) \mathrm{d} s & =\frac{\dot{V}_{0}(t)}{V_{0}^{2}(t)} \int_{t-s_{-}}^{t}(t-s) \dot{v}_{i 0}(s) \mathrm{d} s \\
& =\frac{\dot{V}_{0}(t)}{V_{0}^{2}(t)}\left[-s_{-} v_{i 0}\left(t-s_{-}\right)+\int_{t-s_{-}}^{t} v_{i 0}(s) \mathrm{d} s\right] .
\end{aligned}
$$


The quantities $\mathcal{K}_{0}(s)$ and $\mathbf{m}_{0}(s) \cdot \nabla d_{1}(s)$ have been removed from the integration by the same argument as used in (4.33). We have

$$
\int_{t-s_{-}}^{t} v_{i 0}(s) \mathrm{d} s=\frac{\epsilon}{V_{0}(t)}\left[h_{i 0}(t) u_{0}(t)-h_{i 0}\left(t-s_{-}\right) u_{0}\left(t-s_{-}\right)\right]-I_{i}(r, t), \quad i=l, s
$$

by virtue of $(4.31)_{1}$ and (4.32), where

$$
I_{i}(r, t)=\int_{t-s_{-}}^{t} h_{i 0 r}(s) u_{0}(s) \mathrm{d} s .
$$

Also

$$
\int_{t-s_{-}}^{t} v_{i 0 r}(s) \mathrm{d} s=\frac{\epsilon}{V_{0}(t)}\left[v_{i 0}(t)-v_{i 0}\left(t-s_{-}\right)\right], \quad i=l, s,
$$

which follows from (4.32). Using $(4.44)_{2}$, we see that

$$
\begin{aligned}
\int_{t-s_{-}}^{t} v_{i 1 r}(s) \mathrm{d} s= & \frac{\epsilon}{V_{0}(t)} \int_{t-s_{-}}^{t} \dot{v}_{i 1}(s) \mathrm{d} s-\frac{\delta(t) \epsilon}{\left(V_{0}(t)\right)^{2}} \int_{t-s_{-}}^{t} \dot{v}_{i 0}(s) \mathrm{d} s-\frac{\epsilon}{\left(V_{0}(t)\right)^{2}} \sum_{j=1}^{2} V_{0 j}(t) \int_{t-s_{-}}^{t}\left(h_{i 0}(s) u_{0}(s)\right)_{\zeta_{j}} \mathrm{~d} s \\
& -\frac{\epsilon}{\left(V_{0}(t)\right)^{2}} \int_{t-s_{-}}^{t}\left(h_{i 0}(s) u_{0}(s)\right)_{t} \mathrm{~d} s+\frac{1}{V_{0}(t)} \sum_{j=1}^{2} V_{0 j}(t) \int_{t-s_{-}}^{t}\left(h_{i 0 r}(s) u_{0}(s)\right)_{\zeta_{j}} \mathrm{~d} s \\
& +\frac{1}{V_{0}(t)} \int_{t-s_{-}}^{t}\left(h_{i 0 r}(s) u_{0}(s)\right)_{t} \mathrm{~d} s, \quad i=l, s
\end{aligned}
$$

where (4.32) has been repeatedly used, along with partial integrations. Also, the dot on the final bracket in some terms implies the full time derivative of the quantity in brackets. The removal of $V_{0}, V_{0 i}$ and $\delta$ from the integrations is justified on the basis of (4.33).

The quantities $u_{0 r}\left(t-s_{-}\right)$and $v_{i 0}\left(t-s_{-}\right)$which arise in (4.45), (4.47), (4.49) and (4.50) can be neglected by virtue of (4.9). The same applies to $u_{0 r}(t)$ and $v_{i 0}(t)$ at large $|r|$.

We now take the difference of (4.45) at $r= \pm \infty$. The quantities $u_{0}\left(t-s_{-}\right), u_{0 \zeta_{i}}\left(t-s_{-}\right), i=1,2$, are not negligible but cancel on taking this difference, because they are independent of $r$ by virtue of the definition of $s_{-}$as introduced in (4.22). These quantities are evaluated at the liquid boundary of $\mathcal{Z}$, a fixed perpendicular distance $r_{-}$from $\Sigma(t)$. Thus, if we choose two positions $\mathbf{x}_{1}$ and $\mathbf{x}_{2}$ on the same perpendicular to $\Sigma(t)$ then $r\left(\mathbf{x}_{1}, t\right)=r_{1}, r\left(\mathbf{x}_{2}, t\right)=r_{2}$ are two different values of $r$. However,

$$
r\left(\mathbf{x}_{1}, t-s_{-}\left(\mathbf{x}_{1}, t\right)\right)=r\left(\mathbf{x}_{2}, t-s_{-}\left(\mathbf{x}_{2}, t\right)\right)=r_{-} .
$$

The $\epsilon$ dependence of $s_{-}$is omitted by virtue of the arguments after (4.42). The same argument in fact applies to $u_{0 r}\left(t-s_{-}\right)$, though this is in any case negligible.

Noting (4.3) and (4.17) 3 , we see that (4.21) means that, to leading order, we can neglect all time dependence except that which occurs through $r(\mathbf{x}, t)$. Thus, we can change variables from $s$ to $r$ in (4.48), observing that, from $(4.17)_{3}, \mathrm{~d} s=\epsilon \mathrm{d} r / V_{0}$, while, as observed above, $t-s_{-}$corresponds to $r_{-}$. Thus,

$$
I_{i}(r, t)=\frac{\epsilon}{V_{0}} \int_{r_{-}}^{r} h_{i 0 r}\left(r^{\prime}\right) u_{0}\left(r^{\prime}, \zeta, t\right) \mathrm{d} r^{\prime}, \quad i=l, s
$$

where the fact that $\varphi_{0}$ and therefore $h_{i 0}$ depends only on $r$ has been incorporated. Recalling the observation after (4.6), we see that $r_{-}$is a large negative quantity, which to leading order, can be replaced by $-\infty$. Therefore, $I_{i}(-\infty, t)$ is negligible and

$$
I_{s}(\infty, t)=\frac{\epsilon}{V_{0}} u_{a}(\zeta, t), \quad I_{l}(\infty, t)=-I_{s}(\infty, t)
$$

where $u_{a}$ is defined by $(4.37)_{2}$ and given by the generalization of (3.56) to the present context (see after (4.38)). Similar observations apply to the last two terms of (4.50).

The integral term over points outside of $\mathcal{Z}$ is neglected on the grounds that $\left[\Delta \bar{u}_{0}^{t}\right]$ will be non-zero only for a portion of the integration of order $s_{-}$or $\epsilon$, as argued in relation to (3.71).

Recalling (4.8), we obtain, putting $\left[\bar{u}_{0 \zeta_{i}}\right]=\left[\bar{u}_{0}\right]_{\zeta_{i}}$ and $\left[\bar{u}_{0 t}\right]=\left[\bar{u}_{0}\right]_{t}$,

$$
\begin{aligned}
\left(V_{0}^{2}-V_{s}^{2}\right) u_{1 r}(\infty)-\left(V_{0}^{2}-V_{l}^{2}\right) u_{1 r}(-\infty) & =\left(V_{0}^{2}-V_{s}^{2}\right) \bar{u}_{0 d}(0+)-\left(V_{0}^{2}-V_{l}^{2}\right) \bar{u}_{0 d}(0-) \\
& =\left(V_{0}^{2}-V_{+}^{2}\right)\left[u_{1 r}\right]_{-}-V_{-}^{2}\left[u_{1 r}\right]_{+}=\left(V_{0}^{2}-V_{+}^{2}\right)\left[\bar{u}_{0 d}\right]_{-}-V_{-}^{2}\left[\bar{u}_{0 d}\right]_{+} \\
& =\frac{1}{V_{0}}\left\{-\left(V_{0}^{2}+V_{J}^{2}\right)\left(\sum_{j=1}^{2} V_{0 j}\left[\bar{u}_{0}\right]_{\zeta_{j}-}+\left[\bar{u}_{0}\right]_{t-}\right)+C_{J}\left[u_{0}\right]_{-}\right\}
\end{aligned}
$$

noting that the quantities involving $\varphi$ vanish. The first three relations provide different forms of the same expression. The quantities $V_{+}^{2}$ $V_{J}^{2}$ are given by (3.44) and (3.59) $)_{2}$ respectively, while $C_{J}$ is defined by

$$
\begin{aligned}
& C_{J}=(1-J) C_{s}+J C_{l} \\
& C_{i}=k_{i}^{\prime}(0)+k_{i}(0)\left(\frac{\dot{V}_{0}}{V_{0}}-V_{0} \mathcal{K}_{0}\right), \quad i=l, s .
\end{aligned}
$$


Note the extra term proportional to $\dot{V}_{0}(t)$ which does not occur in the steady-state case as given by (3.74) at $s=0$. Also

$$
\frac{1}{V_{0}} C_{J}\left[u_{0}\right]_{-}=\frac{C_{J} V_{0} \gamma\left(\mathbf{V}_{0}\right)}{\left(V_{0}^{2}-V_{J}^{2}\right)}
$$

by virtue of (4.39). All quantities in (4.54) are evaluated at $(\zeta, t)$ where $\zeta$ is the local tangential coordinate system with origin $\mathbf{x}_{0}$. For a solidification process, it is understood that $V_{0}$ is positive.

Comparing with (3.75), we see that the first term on the left is not necessarily negligible. If however, $V_{0 i} / V_{0}, i=1,2$ and $\left[\bar{u}_{0}\right]_{t-} / V_{0}$ are small or if $\left[\bar{u}_{0}\right]_{\zeta_{i}-}, i=1,2,\left[\bar{u}_{0}\right]_{t-} / V_{0}$ are small then this term may be neglected.

The relations (3.78)-(3.81) can readily be adapted to the present, more general context. The discussion on the power behaviour of $\gamma(\mathbf{V}),\left[\bar{u}_{0}\right]_{-}$etc. following these relations also applies in this more general case. We refer to a detailed discussion of this topic in ([1], end of Subsection 6.1).

By reversing the sign of $V_{0}$ and $\mathcal{K}_{0}$, relations (4.39) and (4.54) apply also to the liquefaction case as discussed and the end of Section 3.2. This applies also to the extra terms not included in the earlier discussion.

\subsection{Outside of the transition zone}

The outer expansions are relevant for purposes of matching the inner expansions. They need not be introduced when considering (2.19) outside of $\mathcal{Z}$. Indeed, we see from (4.10) and (4.11) that the $\varphi$ dependence drops out completely.

In the ablating, liquid phase, we have the property that if $\mathbf{x} \in \mathscr{B}_{\beta}$ at time $t$, it has this property at all earlier times. ${ }^{6}$ Thus, the relevant equation (cf. (3.82))

$$
\dot{u}(\mathbf{x}, t)=k \circ \Delta u^{t}(\mathbf{x}), \quad \mathbf{x} \in \mathscr{B}_{\beta}
$$

is well-defined. The situation is more complicated when $\mathbf{x} \in \mathcal{B}_{\alpha}$. Let us assume that $\mathbf{x} \in \Sigma(t-\tau(\mathbf{x}, t))$, where the surface $\Sigma$ is defined after (2.26). Thus, the reference interface passed over $\mathbf{x}$ at a time $t-\tau$. Starting from (2.21), the developments from (3.93)-(3.104) can be generalized. Just as $(3.98)_{2}$ corresponds to the leading term in (3.35), the analogous term here corresponds to the expression on the left of (4.34), without the differentiation with respect to $r$. We obtain

$$
\mathbf{q}(\mathbf{x}, t)=-k_{s} \circ \nabla u^{t}(\mathbf{x}, \tau(\mathbf{x}, t))-k_{l} \circ \nabla u^{t}(\mathbf{x}, \tau(\mathbf{x}, t), \infty)-k_{J}(\tau(\mathbf{x}, t)) \nabla \tau(\mathbf{x}, t)\left(u^{t}\left(\mathbf{x}, \tau_{-}(\mathbf{x}, t)\right)-u^{t}\left(\mathbf{x}, \tau_{+}(\mathbf{x}, t)\right)\right)
$$

where $\tau_{-}(\mathbf{x}, t)$ is the limit $s \rightarrow \tau(\mathbf{x}, t)$ from below and $\tau_{+}(\mathbf{x}, t)$ is this limit from above. We take $\Sigma(t-\tau)$ as the surface of discontinuity of $u$, after the developments just referred to, which determine the final term on the right of (4.58). In other words, the transition zone structure is neglected in the final equations. The quantity $k_{J}$ is defined by $(3.101)_{2}$. Also

$$
-\nabla \cdot \mathbf{q}(\mathbf{x}, t)=\dot{u}(\mathbf{x}, t)=k_{s} \circ \Delta u^{t}(\mathbf{x}, \tau(\mathbf{x}, t))+k_{l} \circ \Delta u^{t}(\mathbf{x}, \tau(\mathbf{x}, t), \infty)+j(\mathbf{x}, t)
$$

with

$$
\begin{aligned}
j(\mathbf{x}, t)= & \left(k_{J}^{\prime}(\tau)|\nabla \tau|^{2}+k_{J}(\tau) \Delta \tau\right)\left(u^{t}\left(\mathbf{x}, \tau_{-}(\mathbf{x}, t)\right)-u^{t}\left(\mathbf{x}, \tau_{+}(\mathbf{x}, t)\right)\right) \\
& +\nabla \tau(\mathbf{x}, t) \cdot\left(\left.k_{s}(\tau) \nabla u^{t}(\mathbf{x}, s)\right|_{s=\tau_{-}(\mathbf{x}, t)}-\left.k_{l}(\tau) \nabla u^{t}(\mathbf{x}, s)\right|_{s=\tau_{+}(\mathbf{x}, t)}\right),
\end{aligned}
$$

where, as in (3.107), we have neglected terms involving $\nabla u^{t}\left(\mathbf{x}, \tau_{ \pm}(\mathbf{x}, t)\right)$, where $\tau_{ \pm}(\mathbf{x}, t)$ is included in the differentiation, since varying $\mathbf{x}$ in these quantities will give $u^{t}$ at nearly equal positions on the surface $\Sigma(t-\tau)$ of constant $\varphi$. By virtue of the straightforward adaption of (3.53) to the general case, $u^{t}$ will vary negligibly over this surface.

We can write $j$ evaluated at $\left(\mathbf{x}_{-}, \tau(\mathbf{x}, t)\right)$ and $\left(\mathbf{x}_{+}, \tau(\mathbf{x}, t)\right)$ where $\left(\mathbf{x}_{+}, \tau\right)$ is on the same side of the discontinuity as $\left(\mathbf{x}, \tau_{+}\right)$. Similarly for $\mathbf{x}_{-}$and $\tau_{-}$. Then $j$ can be expressed in terms of $\left[\bar{u}_{0}\right]$ given by (4.39) evaluated at time $t-\tau$.

Relation (4.59) is the form of the heat equation in the solid zone. Note that $u^{t}\left(\mathbf{x}, s^{\prime}\right), s^{\prime}>\tau$ which occurs in the second term on the right of (4.59), is in fact the field variable in the liquid zone, given as a solution of (4.57), at time $(t-\tau(\mathbf{x}, t))$ or earlier. This interrelated system of integro-partial differential equations (4.57) and (4.59) must be subject to suitable initial and boundary conditions.

These equations are mainly of interest in situations where information on the shape and evolution of the transition zone is supplied. They correspond to the classical Stefan problem, which is known to be very difficult to tackle if $\mathcal{Z}$ is not given but has to be deduced. For the above equations, this difficulty would no doubt be very much greater.

This was a major motivation for the development of the phase field approach. If information on $\mathcal{Z}$ must be deduced, the only practical approach is a (no doubt) numerical solution of the system (2.19), where the transition zone is assigned a finite width.

\section{References}

[1] J.M. Golden, Phase transitions in materials with thermal memory, Physica D 237 (2008) 2499-2516.

[2] E. Fried, M.E. Gurtin, Continuum theory of thermally induced phase transitions based on an order parameter, Physica D 68 (1993) $326-343$.

[3] E. Fried, M.E. Gurtin, A phase-field theory for solidification based on a general anisotropic sharp-interface theory with interfacial energy and entropy, Physica D 91 (1996) $143-181$.

[4] M.E. Gurtin, A.C. Pipkin, A general theory of heat conduction with finite wave speeds, Arch. Ration. Mech. Anal. 31 (1968) 113-126.

[5] M. Fabrizio, C. Giorgi, A. Morro, A thermodynamic approach to non-isothermal phase-field evolution in continuum physics, Physica D 214 (2006) $144-156$.

[6] G. Caginalp, P.C. Fife, Dynamics of layered interfaces arising from phase boundaries, SIAM J. Appl. Math. 48 (1988) 506-518.

[6] G. Caginalp, P.C. Fife, Dynamics of layered interfaces arising from phase boundaries, SIAM J. Appl. Math. 48 (1988

[8] G.B. McFadden, A.A. Wheeler, D.M. Anderson, Thin interface asymptotics for an energy/entropy approach to phase-field models with unequal conductivities, Physica D 144 (2000) $154-168$.

[9] J.M. Golden, G.A.C. Graham, Boundary Value Problems in Linear Viscoelasticity, Springer, Berlin, 1988.

6 This observation plays a basic role in another context, that of viscoelastic boundary value problems [9]. 